\title{
SHOCKED POSTSTARBURST GALAXY SURVEY. II. THE MOLECULAR GAS CONTENT AND PROPERTIES
} OF A SUBSET OF SPOGs

\author{
Katherine Alatalo ${ }^{1,8}$, Ute Lisenfeld ${ }^{2}$, Lauranne Lanz $^{3}$, Philip N. Appleton ${ }^{3}$, Felipe Ardila ${ }^{4}$, Sabrina L. Cales ${ }^{5}$, \\ Lisa J. Kewley ${ }^{6}$, Mark Lacy ${ }^{7}$, Anne M. Medling ${ }^{6}$, Kristina Nyland ${ }^{7}$, Jeffrey A. Rich ${ }^{1,3}$, And C. Meg Urry ${ }^{5}$ \\ ${ }^{1}$ Observatories of the Carnegie Institution of Washington, 813 Santa Barbara Street, Pasadena, CA 91101, USA; kalatalo@ carnegiescience.edu \\ ${ }^{2}$ Departamento de Física Teórica y del Cosmos, Universidad de Granada, Granada, Spain \\ ${ }^{3}$ Infrared Processing and Analysis Center, California Institute of Technology, Pasadena, CA 91125, USA \\ ${ }^{4}$ Department of Astrophysical Sciences, Princeton University, Peyton Hall, 4 Ivy Lane, Princeton, NJ 08544, USA \\ ${ }^{5}$ Department of Astronomy, Yale University, New Haven, CT 06511, USA \\ ${ }^{6}$ Research School of Astronomy and Astrophysics, Australian National University, Cotter Road, Weston ACT 2611, Australia \\ ${ }^{7}$ National Radio Astronomy Observatory, 520 Edgemont Road, Charlottesville, VA 22903, USA \\ Received 2016 February 6; revised 2016 May 19; accepted 2016 June 2; published 2016 August 11
}

\begin{abstract}
We present $\mathrm{CO}(1-0)$ observations of objects within the Shocked POststarburst Galaxy Survey taken with the Institut de Radioastronomie Millimétrique $30 \mathrm{~m}$ single dish and the Combined Array for Research for Millimeter Astronomy interferometer. Shocked poststarburst galaxies (SPOGs) represent a transitioning population of galaxies, with deep Balmer absorption $\left(\mathrm{EW}_{\mathrm{H} \delta}>5 \AA\right.$ ), consistent with an intermediate-age (A-star) stellar population, and ionized gas line ratios inconsistent with pure star formation. The $\mathrm{CO}(1-0)$ subsample was selected from SPOGs detected by the Wide-field Infrared Survey Explorer with $22 \mu \mathrm{m}$ flux detected at a signal-to-noise ratio $(\mathrm{S} / \mathrm{N})>3$. Of the 52 objects observed in $\mathrm{CO}(1-0), 47$ are detected with $\mathrm{S} / \mathrm{N}>3$. A large fraction $(37 \%-$ $46 \% \pm 7 \%$ ) of our CO-SPOG sample were visually classified as morphologically disrupted. The $\mathrm{H}_{2}$ masses detected were between 10 $0^{8.7-10.8} M_{\odot}$, consistent with the gas masses found in normal galaxies, though approximately an order of magnitude larger than the range seen in poststarburst galaxies. When comparing the $22 \mu \mathrm{m}$ and $\mathrm{CO}(1-0)$ fluxes, SPOGs diverge from the normal star-forming relation, having $22 \mu \mathrm{m}$ fluxes in excess of the relation by a factor of $\left\langle\epsilon_{\mathrm{MIR}}\right\rangle=4.91_{-0.39}^{+0.42}$, suggestive of the presence of active galactic nuclei (AGNs). The $\mathrm{Na}$ I D characteristics of CO-SPOGs show that it is likely that many of these objects host interstellar winds. Objects with large $\mathrm{Na} I \mathrm{D}$ enhancements also tend to emit in the radio, suggesting possible AGN driving of neutral winds.
\end{abstract}

Key words: galaxies: active - galaxies: evolution - galaxies: interactions - galaxies: ISM - galaxies: star formation - radio lines: galaxies

\section{INTRODUCTION}

The bimodality of morphological classifications of galaxies has long been known (Hubble 1926). Typical galaxies are classified as either "late-type" galaxies or "early type" galaxies. "Late types" have thin disks and exhibit spiral structure and blue colors. "Early types" tend to be more ellipsoidal, contain smoother isophotes, and exhibit redder colors. Galaxies also bifurcate across colors with a red and blue population (Baade 1958; Holmberg 1958; Tinsley 1978; Strateva et al. 2001; Baldry et al. 2004; Faber et al. 2007) based primarily on their star formation properties, and few galaxies have intermediate, "green valley" colors (Bell et al. 2003). The morphological and color bimodalities seem to indicate that galaxies transform rapidly between the blue cloud and red sequence (Martin et al. 2007). Star-forming galaxies are blue in color and span a large range of magnitudes, known as the "blue cloud." Red sequence galaxies, on the other hand, inhabit a well-defined region with much smaller variation in both color and magnitude. As in the case of the morphological classification of galaxies, the color bimodality seen in galaxies can be explained simply by quenching star formation. Once a star-forming galaxy has had its star formation quenched, it quickly migrates from the blue cloud and becomes a red sequence galaxy (Harker et al. 2006). The morphological and color properties of individual galaxies are

\footnotetext{
${ }^{8}$ Hubble Fellow.
}

usually well matched, with early types also being red sequence galaxies, and late-types also being star-forming galaxies.

Many transformational paths have been proposed, including a merger between two late-type galaxies into an early type in simulation (Toomre \& Toomre 1972; Springel et al. 2005), ram pressure stripping due to falling into a galaxy cluster (Bekki et al. 2002; Park et al. 2007; Blanton \& Moustakas 2009; Chung et al. 2009; Kenney et al. 2014), morphological quenching (Martig et al. 2009, 2013), tidal disruption and harassment through group interactions (Zabludoff \& Mulchaey 1998; Hickson et al. 1992; Rasmussen et al. 2008; Bitsakis et al. 2010, 2014), and active galactic nucleus (AGN) feedback (Fischer et al. 2010; Feruglio et al. 2010, 2015; Alatalo et al. 2011; Sturm et al. 2011; Aalto et al. 2012a, 2012b; Cicone et al. 2012, 2014; Alatalo 2015). In the modern universe $(z \sim 0)$, this transformation appears to be one way (Appleton et al. 2014; Young et al. 2014). Thus, it is essential to understand all pathways that can lead a blue late-type galaxy to become a red early type.

Schawinski et al. (2014) showed that the majority of galaxies with green colors were in fact normal spiral galaxies, with normal star formation rates that had built up a significant population of intermediate-age and older stars. For this reason, optical color selection alone is not able to definitively identify a transitioning galaxy. With the onset of the Wide-field Infrared Survey Explorer (WISE; Wright et al. 2010) mission, evidence mounted that mid-IR colors could be used to identify transitioning galaxies. Alatalo et al. (2014a) presented 
the existence of a prominent bifurcation between star-forming spiral galaxies and quiescent early-type galaxies in the WISE [4.6]-[12] $\mu \mathrm{m}$ bands, deeming this the "Infrared Transition Zone" (IRTZ). Early-type galaxies that were within this IRTZ were found to have red optical colors (also described in Ko et al. 2013; Yesuf et al. 2014), indicating that galaxies must traverse the optical green valley before the IRTZ.

Searches aiming to identify recently quenched galaxies have focused on objects with deep Balmer absorption lines, consistent with the presence of intermediate-age A stars (Cannon \& Pickering 1918), stellar population (Vazdekis et al. 2010), and a lack of ionized gas emission lines usually associated with star formation (nebular lines such as $\mathrm{H} \alpha$ or [O II] $\lambda 3727$; Dressler \& Gunn 1983; Zabludoff et al. 1996; Quintero et al. 2004; Goto 2005, 2007). While this selection is able to find recently quenched galaxies, it provides an incomplete picture of transforming objects. Traditional "E $+\mathrm{A}$ " or " $\mathrm{K}+\mathrm{A}$ " searches miss objects that exhibit line emission, including AGNs (Wild et al. 2009; Cales et al. 2011, 2013; Kocevski et al. 2011), emission from postasymptotic giant branch (post-AGB) stars (Yan et al. 2006), and shocks (Allen et al. 2008; Rich et al. 2011; Alatalo et al. 2016). Traditional poststarburst searches also miss galaxies that have quenched but still have some low-level $\mathrm{H} \alpha$ or [O II] emission (Yesuf et al. 2014; Rowlands et al. 2015).

While the most common picture of galaxy evolution involves the exhaustion of the star-forming fuel prior to the complete cessation of star formation (Hopkins et al. 2006), observations of molecular gas in "red and dead" galaxies seem to indicate that a molecular reservoir can remain intact after galaxy transformation (Combes et al. 2007; Davis et al. 2011; Young et al. 2011), even without re-accretion of new molecular material, although in these cases the molecular gas mass is no more than $1 \%$ of the total stellar mass. More recently, French et al. (2015) and Rowlands et al. (2015) have been able to show that significant reservoirs of molecular gas remain in post-transition objects (in these cases, poststarburst galaxies), calling into question the need to completely deplete (or significantly reduce) the molecular reservoir within a galaxy to cause star formation quenching and galaxy transition.

This reservoir of gas could also be explained if poststarburst galaxies originate when early-type galaxies accrete material from the environment and go through a brief starburst phase, which was suggested by recent observations of a large sample of galaxies by Dressler et al. (2013). Recently, interferometric molecular gas observations have shown that the star formation within some quenched objects is suppressed, with inefficiencies of factors of 20-70 (Aalto et al. 2016; Alatalo et al. 2015a, 2015c; Guillard et al. 2015; Lanz et al. 2016; Salomé et al. 2016), leading to the possibility that star-formationsuppressed molecular reservoirs are a common part of galaxy transformation. However, a larger sample of galaxies must be studied to determine whether this occurs only in rare and energetic objects.

The Shocked POststarburst Galaxy Survey (SPOGS; Alatalo et al. 2016) ${ }^{9}$ was created to search for rapidly transitioning galaxies that would be missed by poststarburst searches, aiming to identify galaxies that are quenching (rather than simply fading; Schawinski et al. 2014). The SPOG sample was drawn

\footnotetext{
http://www.spogs.org
}

from the Oh-Sarzi-Schawinski-Yi sample (OSSY; Oh et al. 2011), selecting only galaxies with bright emission in all diagnostic lines (Baldwin et al. 1981; Veilleux \& Osterbrock 1987), to create the parent emission-line galaxy (ELG) sample (Alatalo et al. 2016). The SPOGS criteria were applied to the ELG sample to include strong Balmer absorption $(\mathrm{EW}(\mathrm{H} \delta)>5 \AA)^{10}$ and ionized gas emission that is inconsistent with pure star formation. While SPOGS is by no means complete, these criteria have resulted in selecting 1067 candidate objects (deemed SPOGs). Further details of the SPOGS are available in Alatalo et al. (2016).

We present new Combined Array from Research in Millimeter Astronomy (CARMA) and Institut de RAdioastronomie Millimétrique (IRAM) $30 \mathrm{~m} \mathrm{CO(1-0)} \mathrm{observations}$ of 52 SPOGs. In Section 2, we describe the selection used to draw the $\mathrm{CO}(1-0)$ sample. In Section 3, we describe the observations from IRAM and CARMA, including reduction and analysis methods. In Section 4, we present the molecular properties of the sample. In Section 5, we discuss these results in the context of transitioning galaxies. In Section 6, we summarize our results. The cosmological parameters $H_{0}=70 \mathrm{~km} \mathrm{~s}^{-1}, \quad \Omega_{\mathrm{m}}=0.3 \quad$ and $\quad \Omega_{\Lambda}=0.7 \quad$ (Spergel et al. 2007) are used throughout.

\section{THE CO(1-0) SAMPLE}

The objects chosen for $\mathrm{CO}(1-0)$ observations were selected based on the SPOG subsample cross-correlated with the WISE All-sky Survey (Wright et al. 2010), detailed in Alatalo et al. (2014a), to have detectable (signal-to-noise ratio [S/N] > 3) $22 \mu \mathrm{m}$ fluxes. The $22 \mu \mathrm{m}$ emission is usually associated with star formation (Calzetti et al. 2007), but it is also strong in AGNs (Ward et al. 1987; Sanders et al. 1989; Elvis et al. 1994). The $22 \mu \mathrm{m}$ emission can also arise in quiescent galaxies, from dust that is heated by the aging stellar population (Draine et al. 2007; Crocker et al. 2011, 2013; Dale et al. 2012). Because our SPOG selection criteria remove galaxies whose ionized gas line ratios are dominated by star formation, the $22 \mu \mathrm{m}$ emission in these sources is less likely to be associated primarily with star formation. It is possible that some of our objects are in fact "skin effect contaminants," in which the bulk of star formation is obscured from the optical view (in a compact core), and in which the overlying material is heated primarily by older stellar populations (Wild et al. 2011). This cannot be ruled out completely until other star formation indicators have been measured. Despite this, it is unlikely that this is a dominant effect, as in the vast majority of cases, the optical emission and ionized gas line ratios of dusty, buried starbursts manifest as star-forming (Casey et al. 2014; Rich et al. 2015). A caveat to this is that this scenario cannot remove star-forming objects completely and thus does not remove objects in which the dominant star formation is taking place outside of the $3^{\prime \prime}$ SDSS fiber (although this is most problematic at low redshifts, where the fiber only traces the nucleus; Rich et al. 2014; Alatalo et al. 2016).

Of the 1067 SPOGs, $491(46 \% \pm 2 \%)$ are detected with an $\mathrm{S} / \mathrm{N}$ of at least 3 in the WISE $22 \mu \mathrm{m}$ band. Radio identifications of the WISE $22 \mu \mathrm{m}$-detected sample were made via a 1."5 radial match (Ivezić et al. 2002) with the Faint Images of the Radio

\footnotetext{
${ }^{10}$ It is possible that the SPOGS selection has missed shocked galaxies without ongoing star formation based on the requirement for such deep absorption, given the possibility of Balmer emission filling the stellar absorption features.
} 


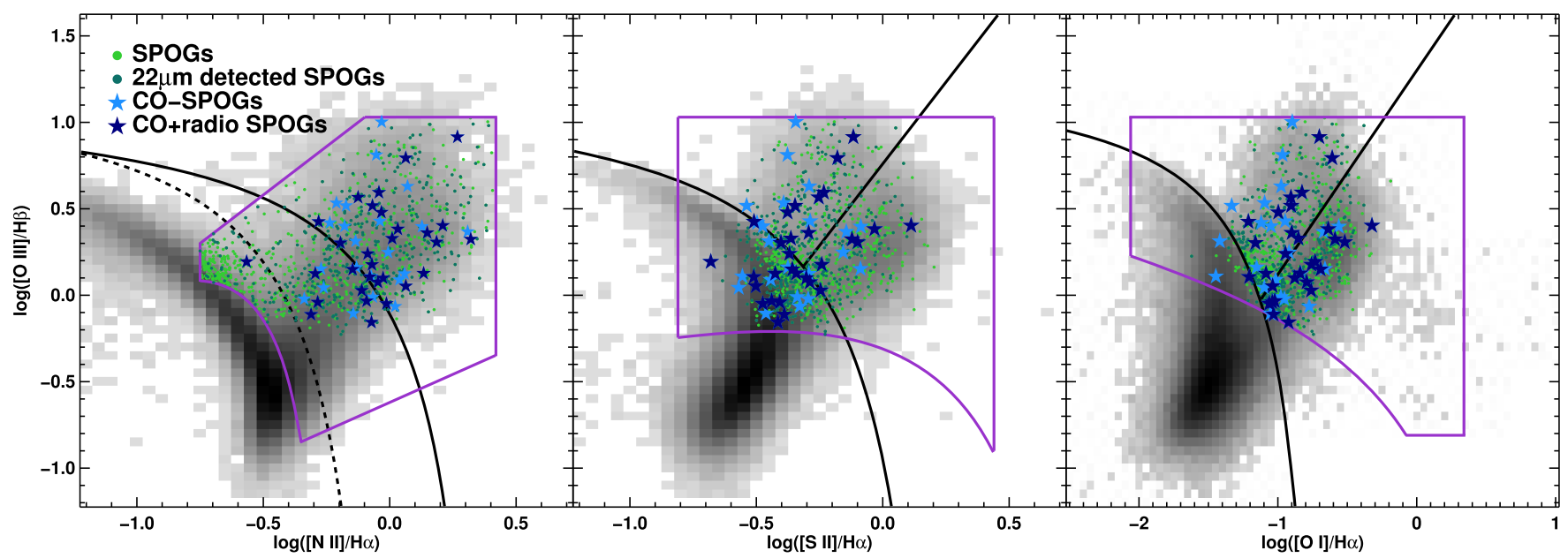

Figure 1. Ionized gas line ratios of the ELG (grayscale) and SPOG samples (green dots; Alatalo et al. 2016), including [N II] $/ \mathrm{H} \alpha$ vs. $[\mathrm{O}$ III] $/ \mathrm{H} \beta$ (left; Baldwin et al. 1981), [S II] $/ \mathrm{H} \alpha$ vs. [O III] $/ \mathrm{H} \beta$ (middle), and [O I] $/ \mathrm{H} \alpha$ vs. [O III] $/ \mathrm{H} \beta$ (right; Veilleux \& Osterbrock 1987), overlaid with the line diagnostic models of Kauffmann et al. (2003) and Kewley et al. (2006). The purple line defines the boundaries of the shock models, SPOG criterion (Allen et al. 2008; Rich et al. 2011; Alatalo et al. 2016). WISE $22 \mu \mathrm{m}$-detected SPOGs are shown in dark green. CO-SPOGs (light blue stars) and $1.4 \mathrm{GHz}$ radio-matched CO-SPOGs (dark blue stars) are also shown. CO-SPOGs span the ionized gas diagnostic space of the larger SPOG sample. There is also little difference between the radio-matched and unmatched $22 \mu$ m-detected SPOGs.

Sky at Twenty Centimeters (FIRST) Survey (Becker et al. 1995), described in Alatalo et al. (2016). There are 83/1067 concurrent WISE $22 \mu \mathrm{m}$ and FIRST-detected objects $(8 \% \pm 1 \%)$, which accounts for over $50 \%$ of the $(160 / 1067)$ total radio matches among the entire SPOG sample.

The IRAM $30 \mathrm{~m}$ sample was selected from the 491 WISE $22 \mu \mathrm{m}$-detected SPOGs. The WISE $22 \mu \mathrm{m}$-detected sample was divided into bins based on radio detections and radio nondetections, as well as shock models within the $[\mathrm{OI}] / \mathrm{H} \alpha$ versus $[\mathrm{O} \mathrm{III}] / \mathrm{H} \beta$ line diagnostic diagram (Allen et al. 2008; see also Figure 1(c)), selecting one radio detection and one nondetection per bin. Of the 40 IRAM $30 \mathrm{~m}$ proposed objects, 35 were observed and 30 were detected. The CARMAobserved SPOG sample focused on a WISE $22 \mu$ m flux-limited sample within the R.A. range of $9^{\mathrm{h}}-15^{\mathrm{h}}$. There were 51 SPOGs with $\mathrm{F}_{22 \mu \mathrm{m}}>14 \mathrm{mJy}$ within this R.A. range that were queued for observation, and 19 were successfully observed. Table 1 presents the general properties of the objects observed with the IRAM $30 \mathrm{~m}$ and CARMA.

Our requirement for a WISE $22 \mu \mathrm{m}$ detection used to construct our follow-up sample of $\mathrm{CO}$ observations may bias this subset of SPOGs to favor those harboring AGNs, an aging stellar population, or dusty compact starbursts. This is supported by the high prevalence of FIRST radio detections, which trace emission from AGNs (although they can also trace star formation; Condon 1992). Figure 1 displays SPOGs (green circles) on the emission-line ratio diagnostic diagrams (Baldwin et al. 1981; Veilleux \& Osterbrock 1987), with $22 \mu$ m-detected sources (sky blue stars) identified, as well as concurrently $22 \mu \mathrm{m}+1.4 \mathrm{GHz}-$ detected objects (dark blue stars). Figure 1 shows that the emission-line ratios of our CO-observed SPOG sample (hereafter CO-SPOGs) do not exclusively lie in the Seyfert ionized gas ratio space or star-forming space (Kewley et al. 2006). A large set of objects in this sample are consistent with the low-ionization nuclear emission-line region (LINER) portion of the diagram. This strongly suggests that the $22 \mu \mathrm{m}$ selection has selected AGNs in the SPOG sample.
To improve accuracy, we calculate the total stellar masses of our CO-SPOGs by fitting the complete (FUV, NUV, u, g, r, i, $\mathrm{z}, \mathrm{J}, \mathrm{H}, \mathrm{K}_{\mathrm{s}}$ ) photometry using MAGPHYS (da Cunha et al. 2008), which takes into account extinction and $k$-corrections. SED fitting of the full SPOG sample will be presented in T. Bitsakis et al. (2016), in preparation.

The selection for the CO-SPOG sample also spans the entire $u-r$ color range of SPOGs (Figure 2), but appears to trace more massive objects than the underlying SPOG population.

Between the IRAM $30 \mathrm{~m}$ and CARMA samples, a total of 52 objects were observed (two objects overlapped between the CARMA and IRAM $30 \mathrm{~m}$ samples, with CARMA detections favored due to better S/N). Figure 3 shows the SDSS gri threecolor thumbnails of all objects, and details of the observations are presented below.

\section{OBSERVATIONS AND ANALYSIS}

\subsection{The IRAM $30 \mathrm{~m}$}

We observed the $\mathrm{CO}(1-0)$ line at the central position of our sample galaxies on 2014 September 9-14, October 15-18, and November 6-9 with the IRAM $30 \mathrm{~m}$ telescope on Pico Veleta. We used the dual-polarization Eight MIxer Receiver (EMIR; Carter et al. 2012) in combination with the autocorrelator Fourier Transform Spectrometers at a frequency resolution of $0.195 \mathrm{MHz}$ at $\mathrm{CO}(1-0)$ (providing a velocity resolution of $0.57 \mathrm{~km} \mathrm{~s}^{-1}$ ). The observations were done in wobbler switching mode with a wobbler throw of $240^{\prime \prime}$ in the azimuthal direction.

The broad bandwidth of the receiver $(16 \mathrm{GHz})$ and backend allowed us to group the observations of galaxies with similar redshifts. The central sky frequencies, taking into account the redshift of the objects, ranged between 96 and $111 \mathrm{GHz}$. Each object was observed until it was detected with an $\mathrm{S} / \mathrm{N}$ of at least 5 or until an rms of $1.5 \mathrm{mK}\left(\mathrm{T}_{\mathrm{A}}^{*}\right)$ was achieved for a velocity resolution of $20 \mathrm{~km} \mathrm{~s}^{-1}$. The integration times per object ranged between 0.5 and $2 \mathrm{hr}$, with a mean value of 80 minutes. Pointing was monitored on nearby quasars every 
Table 1

CO-SPOG Sample Properties

\begin{tabular}{|c|c|c|c|c|c|c|c|c|c|}
\hline & $\begin{array}{l}\text { SPOG } \\
\text { Name } \\
(1)\end{array}$ & $\begin{array}{c}\text { Telescope } \\
\text { (2) }\end{array}$ & $\begin{array}{l}\text { R.A. } \\
\text { (J2000) } \\
(3)\end{array}$ & $\begin{array}{l}\text { Decl. } \\
(\text { J2000) } \\
(4)\end{array}$ & $\begin{array}{l}z \\
(5)\end{array}$ & $\begin{array}{c}\mathrm{F}_{22 \mu \mathrm{m}} \\
(\mathrm{mJy}) \\
(6)\end{array}$ & $\begin{array}{c}\mathrm{F}_{1.4} \\
(\mathrm{mJy}) \\
(7)\end{array}$ & $\begin{array}{c}\log \left(M_{\star}\right) \\
\left(M_{\odot}\right) \\
(8)\end{array}$ & $\begin{array}{c}\text { Morphology } \\
\text { (9) }\end{array}$ \\
\hline 1 & $\mathrm{~J} 0003+0048$ & I & 000318.21 & +004844.2 & 0.139 & $14.2 \pm 1.6$ & $3.71 \pm 0.096$ & 10.72 & $\checkmark$ \\
\hline 4 & J0011-0054 & I & 001145.21 & -005444.2 & 0.048 & $20.5 \pm 1.3$ & $2.08 \pm 0.14$ & 10.34 & $\ldots$ \\
\hline 7 & $\mathrm{~J} 0029+1433$ & I & 002928.96 & +143342.8 & 0.143 & $10.2 \pm 2.0$ & $\ldots$ & 10.69 & $\cdots$ \\
\hline 13 & J0037+0024 & I & 003707.82 & +002436.3 & 0.081 & $21.1 \pm 1.5$ & $\ldots$ & 10.18 & $\checkmark$ \\
\hline 24 & J0119+1334 & I & 011956.76 & +133431.4 & 0.191 & $3.4 \pm 0.3$ & $\ldots$ & 10.95 & $\checkmark$ \\
\hline 77 & $\mathrm{~J} 0803+2530$ & I & 080359.61 & +253051.4 & 0.135 & $11.3 \pm 1.2$ & $1.13 \pm 0.14$ & 10.93 & $?$ \\
\hline 81 & $\mathrm{~J} 0807+2006$ & I & 080724.45 & +200608.2 & 0.066 & $21.2 \pm 1.3$ & $3.48 \pm 0.14$ & 10.39 & $\ldots$ \\
\hline 98 & J0816+1936 & I & 081603.14 & +193643.2 & 0.113 & $12.3 \pm 0.99$ & $\ldots$ & 10.40 & $\ldots$ \\
\hline 142 & J0845+2006 & I & 084545.38 & +200610.4 & 0.123 & $17.7 \pm 2.2$ & $\ldots$ & 10.61 & $\cdots$ \\
\hline 157 & $\mathrm{~J} 0853+0310$ & I & 085356.80 & +031033.6 & 0.129 & $3.7 \pm 0.2$ & $1.25 \pm 0.15$ & 10.88 & $\ldots$ \\
\hline 169 & J0859+1006 & I & 085942.62 & +100643.5 & 0.055 & $82.5 \pm 2.3$ & $2.73 \pm 0.14$ & 10.54 & $\checkmark$ \\
\hline 186 & J0914+3753 & $\mathrm{C}$ & 091407.22 & +37 5309.9 & 0.072 & $29.4 \pm 1.4$ & $2.60 \pm 0.16$ & 10.30 & $\cdots$ \\
\hline 191 & $\mathrm{~J} 0918+4200$ & $\mathrm{C}$ & 091849.99 & +420043.5 & 0.041 & $40.4 \pm 1.5$ & $\ldots$ & 10.30 & $\ldots$ \\
\hline 200 & J0925+0623 & $\mathrm{C}$ & 092518.31 & +062334.0 & 0.076 & $27.0 \pm 1.3$ & $\ldots$ & 10.51 & $?$ \\
\hline 209 & $\mathrm{~J} 0928+0741$ & I & 092819.53 & +074158.5 & 0.105 & $20.4 \pm 1.1$ & $\ldots$ & 10.11 & $\checkmark$ \\
\hline 224 & J0938+1819 & $\mathrm{C}$ & 093819.87 & +181952.6 & 0.089 & $5.3 \pm 0.2$ & $4.52 \pm 0.15$ & 10.65 & $?$ \\
\hline 253 & J0957-0012 & $\mathrm{C}$ & 095749.53 & -001252.6 & 0.033 & $36.0 \pm 2.2$ & $0.86 \pm 0.15$ & 10.0 & $\ldots$ \\
\hline 267 & $\mathrm{~J} 1008+0936$ & $\mathrm{C}$ & 100816.22 & +093616.2 & 0.027 & $35.7 \pm 1.7$ & $\ldots$ & 9.97 & $\cdots$ \\
\hline 268 & $\mathrm{~J} 1008+1916$ & I & 100828.72 & +191619.9 & 0.182 & $14.3 \pm 1.0$ & $2.31 \pm 0.14$ & 10.96 & $\ldots$ \\
\hline 270 & $\mathrm{~J} 1008+5123$ & I & 100847.68 & +512352.8 & 0.156 & $14.1 \pm 1.1$ & $\ldots$ & 10.60 & $\checkmark$ \\
\hline 293 & $\mathrm{~J} 1018+1536$ & I & 101823.97 & +153630.9 & 0.111 & $36.0 \pm 1.5$ & $2.99 \pm 0.15$ & 10.78 & $?$ \\
\hline 305 & $\mathrm{~J} 1026+4340$ & $\mathrm{C}$ & 102653.35 & +434008.4 & 0.105 & $41.9 \pm 1.5$ & $1.30 \pm 0.15$ & 10.25 & $\checkmark$ \\
\hline 308 & $\mathrm{~J} 1028+5736$ & I & 102825.80 & +573609.0 & 0.073 & $10.8 \pm 1.1$ & $1.22 \pm 0.16$ & 10.19 & $\cdots$ \\
\hline 322 & $\mathrm{~J} 1031+0540$ & I & 103134.84 & +054057.3 & 0.163 & $10.6 \pm 1.4$ & $\ldots$ & 10.72 & $\checkmark$ \\
\hline 349 & $\mathrm{~J} 1046+2804$ & I & 104636.52 & +280434.6 & 0.128 & $10.8 \pm 1.3$ & $2.44 \pm 0.13$ & 10.42 & $\checkmark$ \\
\hline 365 & $\mathrm{~J} 1057+0554$ & I & 105751.07 & +055446.8 & 0.054 & $27.7 \pm 1.5$ & $1.82 \pm 0.15$ & 10.06 & $\ldots$ \\
\hline 437 & $\mathrm{~J} 1126+1913$ & $\mathrm{C}$ & 112619.44 & +191329.2 & 0.103 & $48.8 \pm 1.6$ & $3.57 \pm 0.15$ & 10.48 & $\checkmark$ \\
\hline 439 & $\mathrm{~J} 1127+1256$ & $\mathrm{C}$ & 112703.64 & +125655.3 & 0.152 & $11.6 \pm 0.60$ & $2.24 \pm 0.14$ & 10.87 & $\ldots$ \\
\hline 462 & $\mathrm{~J} 1136+2453$ & $\mathrm{C}$ & 113655.20 & +245325.4 & 0.033 & $133.4 \pm 4.98$ & $2.55 \pm 0.14$ & 10.12 & $\ldots$ \\
\hline 470 & $\mathrm{~J} 1139+4631$ & $\mathrm{C}$ & 113939.33 & +463132.1 & 0.174 & $26.2 \pm 1.1$ & $4.95 \pm 0.14$ & 11.05 & $\checkmark$ \\
\hline 498 & $\mathrm{~J} 1153+0930$ & $\mathrm{C}$ & 115341.32 & +093025.5 & 0.139 & $48.5 \pm 2.1$ & $1.56 \pm 0.21$ & 10.75 & $\ldots$ \\
\hline 533 & $\mathrm{~J} 1211+2936$ & I & 121138.23 & +293616.5 & 0.107 & $14.1 \pm 1.1$ & $1.32 \pm 0.13$ & 10.59 & $\ldots$ \\
\hline 547 & $\mathrm{~J} 1216+1904$ & I & 121622.27 & +190442.2 & 0.075 & $11.7 \pm 1.1$ & $5.76 \pm 0.14$ & 10.90 & $\checkmark$ \\
\hline 578 & $\mathrm{~J} 1229+3224$ & $\mathrm{C}$ & 122906.93 & +322417.6 & 0.173 & $29.4 \pm 1.2$ & $2.97 \pm 0.13$ & 11.09 & $?$ \\
\hline 619 & $\mathrm{~J} 1248+5514$ & I & 124822.17 & +551452.0 & 0.083 & $13.1 \pm 0.88$ & $\ldots$ & 10.75 & $\cdots$ \\
\hline 658 & $\mathrm{~J} 1313+0207$ & I & 131352.39 & +020757.3 & 0.030 & $12.9 \pm 1.2$ & $\ldots$ & 10.42 & $\ldots$ \\
\hline 662 & $\mathrm{~J} 1314+2106$ & $\mathrm{C}$ & 131447.61 & +210626.2 & 0.046 & $21.5 \pm 1.8$ & $1.97 \pm 0.13$ & 10.47 & $\ldots$ \\
\hline 663 & $\mathrm{~J} 1315+2437$ & $\mathrm{C}$ & 131503.50 & +24 3707.6 & 0.013 & $725.7 \pm 15.6$ & $32.33 \pm 0.13$ & 10.11 & $\cdots$ \\
\hline 689 & $\mathrm{~J} 1326+1922$ & $\mathrm{C}$ & 132648.12 & +192245.8 & 0.174 & $23.1 \pm 1.3$ & $2.09 \pm 0.14$ & 10.89 & $\checkmark$ \\
\hline 704 & $\mathrm{~J} 1336+3008$ & $\mathrm{C}$ & 133604.12 & +300827.9 & 0.026 & $39.4 \pm 2.3$ & $\ldots$ & 9.75 & $\ldots$ \\
\hline 711 & $\mathrm{~J} 1339+4422$ & $\mathrm{C}$ & 133953.18 & +442236.8 & 0.063 & $29.9 \pm 1.1$ & $\ldots$ & 10.47 & $\checkmark$ \\
\hline 745 & $\mathrm{~J} 1356+2816$ & $\mathrm{C}$ & 135643.46 & +281621.3 & 0.133 & $5.1 \pm 0.2$ & $0.99 \pm 0.14$ & 10.79 & $\checkmark$ \\
\hline 767 & $\mathrm{~J} 1409+1016$ & I & 140952.53 & +10 1646.9 & 0.096 & $4.5 \pm 0.2$ & $2.32 \pm 0.15$ & 10.87 & $\checkmark$ \\
\hline 859 & $\mathrm{~J} 1505+5847$ & I & 150541.59 & +584718.9 & 0.145 & $11.1 \pm 0.69$ & $0.85 \pm 0.14$ & 10.80 & $\ldots$ \\
\hline 862 & $\mathrm{~J} 1506+0806$ & I & 150619.17 & +080642.4 & 0.040 & $19.4 \pm 0.89$ & ... & 10.24 & $\cdots$ \\
\hline 908 & $\mathrm{~J} 1529+0601$ & I & 152908.37 & +060119.5 & 0.106 & $18.3 \pm 0.97$ & $\ldots$ & 10.69 & $\ldots$ \\
\hline 909 & $\mathrm{~J} 1529+0913$ & I & 152926.64 & +091325.3 & 0.127 & $11.1 \pm 0.78$ & $\ldots$ & 10.49 & $\checkmark$ \\
\hline 955 & $\mathrm{~J} 1555+2955$ & I & 155524.93 & +295550.8 & 0.070 & $20.6 \pm 0.92$ & $2.96 \pm 0.14$ & 10.32 & $\ldots$ \\
\hline 980 & $\mathrm{~J} 1611+0840$ & I & 161119.39 & +084032.5 & 0.166 & $11.7 \pm 1.1$ & $\ldots$ & 10.59 & $\ldots$ \\
\hline 1014 & $\mathrm{~J} 1645+3048$ & I & 164503.79 & +304802.1 & 0.059 & $35.2 \pm 1.4$ & $\ldots$ & 9.98 & $\ldots$ \\
\hline 1057 & $\mathrm{~J} 2245+1232$ & I & 224532.76 & +123236.2 & 0.093 & $16.4 \pm 1.2$ & $\ldots$ & 10.70 & $\checkmark$ \\
\hline 1062 & J2326-0114 & I & 232637.22 & -011436.2 & 0.197 & $38.4 \pm 1.7$ & $\ldots$ & 10.83 & $\checkmark$ \\
\hline
\end{tabular}

Note. Column (1): SPOG name. Column (2): telescope (I: IRAM 30 m, C: CARMA). Columns (3) and (4): SDSS R.A./decl. Column (5): SDSS redshift. Column (6): $22 \mu \mathrm{m}$ flux from WISE detections. Column (7): $1.4 \mathrm{GHz}$ integrated flux density from FIRST. Column (8): $\log$ of the stellar mass derived using MAGPHYS (da Cunha et al. 2008). Column (9): morphological classification of each CO-SPOG as clearly disrupted $(\checkmark)$, possibly disrupted (?), or not.

60-90 minutes. During the observation period, the weather conditions were generally good, with a pointing accuracy better than $4^{\prime \prime}$. The mean system temperature for the observations was $116 \mathrm{~K}$ on the $T_{\mathrm{A}}^{*}$ scale. At $115 \mathrm{GHz}$, the IRAM forward efficiency, $F_{\text {eff }}$, was 0.95 ; the beam efficiency, $B_{\text {eff }}$, was 0.79 ; and the half-power beam size ranges between 22!" 3 (for $110 \mathrm{GHz}$ ) and 25".6 (for $97 \mathrm{GHz}$ ). All CO spectra and luminosities are presented on the Jansky scale, converted from 


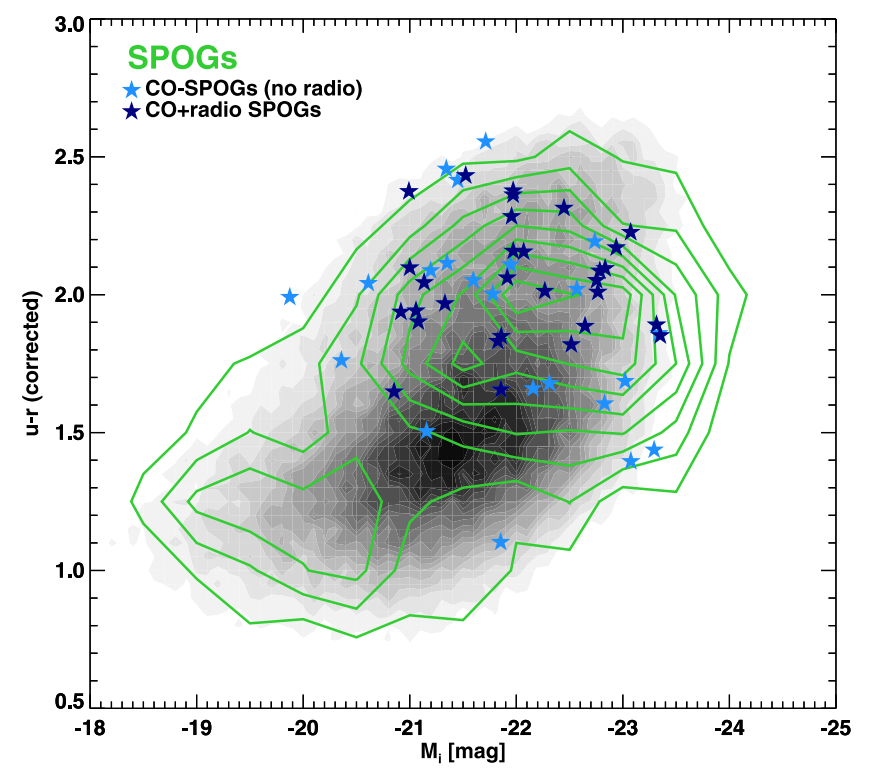

Figure 2. Color-magnitude diagram from the parent emission-line galaxy sample (grayscale; Alatalo et al. 2016) with the distribution of SPOGs overlaid (green contours), with $M_{i}$ representing the (uncorrected) absolute $i$-band magnitude. The CO-SPOGs are overlaid and color-coded based on their radio detections, with FIRST-detected SPOGs shown as dark blue stars, and radio nondetected CO-SPOGs as light blue stars. The CO-SPOGs tend to be more massive than SPOGs in general, but not significantly, and their colors trace those colors of the underlying SPOG distribution fairly well.

the main-beam temperature scale $\left(T_{\mathrm{mb}}\right)$, which is defined as $T_{\mathrm{mb}}=\left(F_{\mathrm{eff}} / B_{\mathrm{eff}}\right) \times T_{\mathrm{A}}^{*}$, using a conversion factor of $5 \mathrm{Jy} \mathrm{K}^{-1}$.

For the data reduction, we first discarded poor scans and then subtracted a constant or linear baseline. A large number of scans were affected by platforming, i.e., the baseline level changed abruptly at one or two positions along the band. This effect could be reliably corrected because the baselines in between these (clearly visible) jumps were flat and allowed to determine the (order 0 or 1) baseline that had to be subtracted from the different parts in order to move the baselines to a zero level along the entire band. After this correction, we summed the spectra of each source, smoothed them to resolutions between 20 and $40 \mathrm{~km} \mathrm{~s}^{-1}$ in order to increase the $\mathrm{S} / \mathrm{N}$ per channel, and visually determined the zero-level widths (the boundaries beyond which the spectra drop to zero). CO-SPOGs with $\mathrm{S} / \mathrm{N}>3$ are considered detected, and CO-SPOGs with $\mathrm{S} / \mathrm{N}>5$ are considered strongly detected. Figure 4 shows the integrated spectra of the 35 SPOGs observed with the IRAM $30 \mathrm{~m}$ (shaded turquoise). The systemic velocity of the line is set to zero, and the total line width is represented by a turquoise line and is shaded in the spectrum. The optically defined systemic velocity is shown as a black dotted line. The velocity-integrated spectra were calculated by summing the individual shaded channels and multiplying by the velocity width of each channel ( 21 or $42 \mathrm{~km} \mathrm{~s}^{-1}$ ).

As an alternative approach, we fit the spectra with a Gaussian profile and integrated it over velocity (details of the Gaussian fitting can be found in Appendix). The velocity-integrated intensities determined by the two methods are in good agreement, with a mean difference of $5 \%$, confirming that our velocity integration is reliable. The line flux rms was then calculated by multiplying the rms per channel by the channel velocity width and the square root of the total number of channels determined to contain line emission (those that are shaded).

\subsection{CARMA}

The CARMA SPOG observations were taken between 2014 June and December using CARMA, an interferometer of 15 radio dishes $(6 \times 10.4$ and $9 \times 6.1 \mathrm{~m})$ located in the Eastern Sierras in California (Bock et al. 2006). ${ }^{11} \mathrm{~A}$ total of 19 SPOGs were observed (with a $100 \%$ detection rate), in either D- or E-array, with baselines in the range $11-150 \mathrm{~m}$ and 8-66 $\mathrm{m}$, respectively. Standard reduction and calibration techniques (as described in detail in Alatalo et al. 2013) were used on all targets.

Our SPOGs were unresolved in all cases except $\mathrm{J} 1315+2437$ (IC 860, which was also observed in CARMA C-array by McBride et al. 2014). The observing parameters associated with each of the CARMA SPOGs are listed in Table 2. A moment0 map was created for each CARMA SPOG (using the MIRIAD task moment; Sault et al. 1995), in which a sigma clip was applied to velocity channels determined to have emission (see Alatalo et al. 2013 for details). We did not apply a standard sigma clip to the channel maps, instead iteratively determining the correct sigma value to maximize the detection of real emission to the exclusion of noise in each individual object. Figure 4 shows the integrated spectra of the 19 SPOGs observed with CARMA (shaded green). The systemic velocity of the line is set to zero, with the total line width represented by a green line, as well as in the shaded region of the spectrum. The integrated spectrum of each SPOG is determined by integrating the flux within an aperture determined by the moment0 map created for each CARMA SPOG.

The rms per channel is calculated by (1) taking the standard deviation of all pixels within the cube that were outside the moment0 aperture, (2) applying an additional noise upcorrection of $30 \%$ to account for the oversampling of the maps (see Alatalo et al. 2015b for details), and (3) multiplying by the square root of the total number of beams represented in the moment 0 aperture.

To calculate the integrated line flux for each galaxy, we summed the channels shaded in green in Figure 4 and multiplied by the velocity width of each channel, listed in Table 2 . The line flux rms was then calculated by multiplying the rms per channel by the channel velocity width and the square root of the total number of channels determined to contain line emission. In the case of the two SPOGs observed by both the IRAM $30 \mathrm{~m}$ and CARMA, the CO fluxes agreed to within the standard $20 \%$ errors.

\section{RESULTS}

\subsection{The Morphologies of CO-SPOGs}

Figure 3 show the three-color gri images from SDSS of all 52 CO-SPOGs. The thumbnails show a large number of galaxies that include signs of interaction, though only a handful appear to be major mergers in the coalescence phase (Dopita et al. 2002). A significant fraction of CO-SPOGs have tidal features prominent enough to be seen in SDSS images. The remaining sample of $\mathrm{CO}-\mathrm{SPOG}$ mainly consists of early-type spirals, lenticular galaxies with bars, and objects with peaked nuclei consistent with AGNs. Our selection did not include morphology, so it is interesting that the number of tidally disrupted objects represented in CO-SPOGs is larger than that in the general SPOG sample.

\footnotetext{
11 http://www.mmarray.org
} 


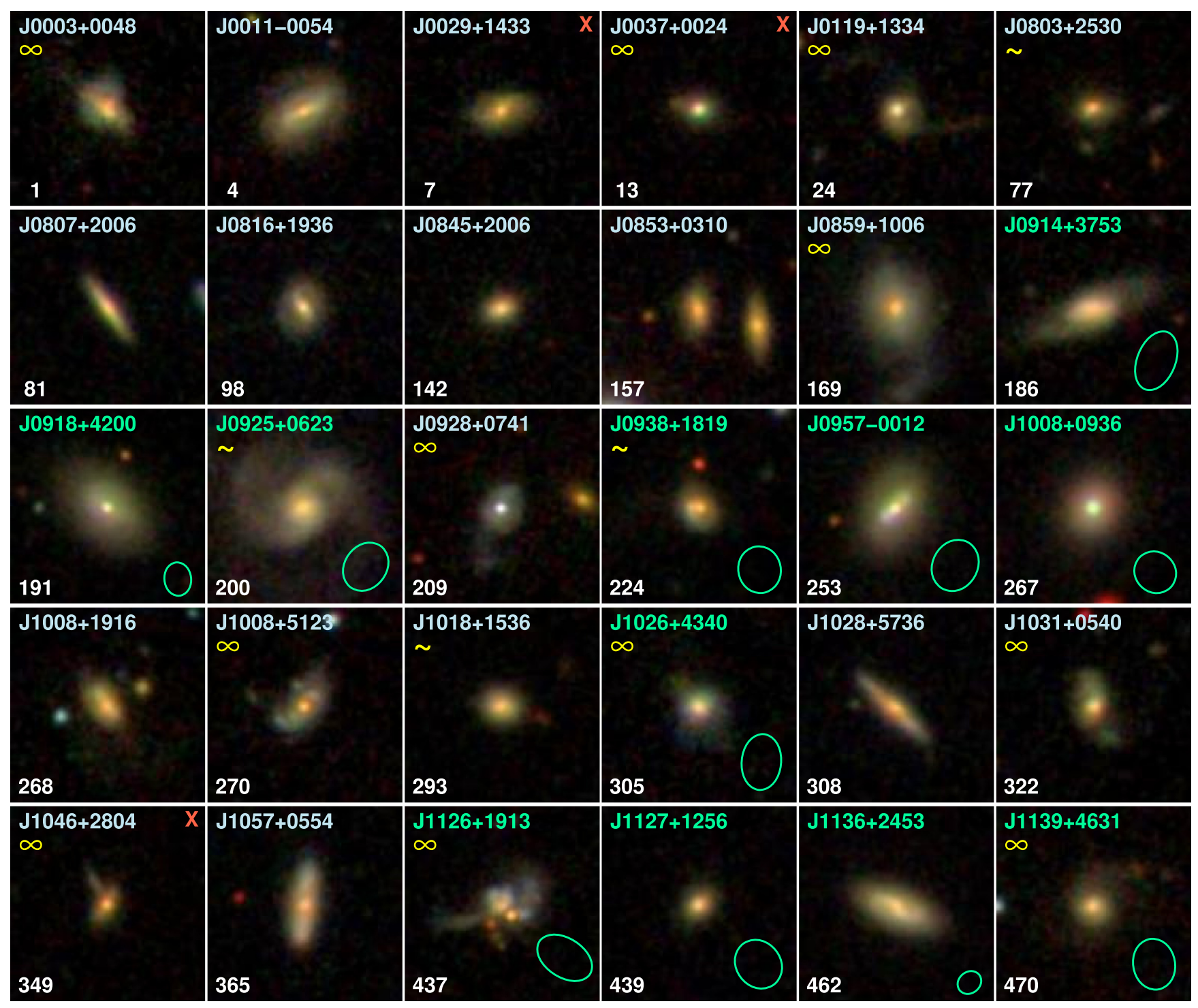

Figure 3. (a) SDSS gri three-color thumbnails of the 52 CO-SPOGs. The field of view of all thumbnails is $30^{\prime \prime}$. Objects detected by CARMA are labeled in green, with the CARMA beam shown as an ellipse in the bottom right. Objects observed with the IRAM $30 \mathrm{~m}$ are labeled in pale blue. The SPOG index from Alatalo et al. (2016) is labeled in white (bottom left). Nondetections are demarcated with a small cross in the top right. Objects that have been morphologically classified as clearly disrupted have a small yellow symbol $(\infty)$ below the object name. Those that are classified as possibly disrupted are demarcated with a $(\sim)$. Many SPOGs show signs of interaction, including tidal tails, dust lanes, and morphological disruption. Most galaxies appear to be red in color, with many also showing signs of peaked, bright nuclei, possibly due to the presence of an AGN. (b) Continuation of the SDSS gri three-color images of CO-SPOGs. The last panel shows the 22"! 3 single-dish primary beam at $\nu_{\mathrm{obs}}=110 \mathrm{GHz}$ for the SPOGs observed with the IRAM $30 \mathrm{~m}$ (labeled in pale blue).

In order to quantify whether the CO-SPOG galaxies are disrupted, six team members (Alatalo, Appleton, Cales, Lanz, Lisenfeld, Nyland) independently visually inspected the gri thumbnails of these galaxies (Figure 3). Based on the (potential) presence of tidally features, they classified the galaxies as (possibly) disrupted or not. There was complete consensus of the classification of 47 galaxies, indicated by the presence or absence of a check mark in Table 1, as well as a $(\infty)$ symbol in Figure 3. The other five galaxies had at least two classifications differing from the others and are therefore marked as ambiguous, represented as a question mark in Table 1 and $(\sim)$ in Figure 3. Based on these classifications, ${ }^{12} 19-24(37 \%-46 \% \pm 7 \%)$ of our CO-SPOGs

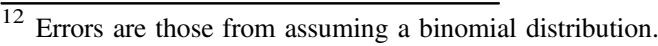

are classified as disrupted. A detailed analysis of the morphologies of SPOGs will be presented in a future paper.

\section{2. $\mathrm{CO}(1-0)$ Properties}

Figure 4 shows the $\mathrm{CO}(1-0)$ spectra of all CO-SPOGs, color-coded to identify which facility was used to make the observation (turquoise for the IRAM $30 \mathrm{~m}$ and green for CARMA). A few double-horned spectral profiles, consistent with molecular gas rotating in a disk that extends out to the flat part of the rotation curve, are present but are the minority of detections (although this could in part be due to sensitivity). In many of the strong molecular gas detections, multiple components and peaks are present in the molecular gas, consistent with the morphological disruption present in gri images of the galaxies. 

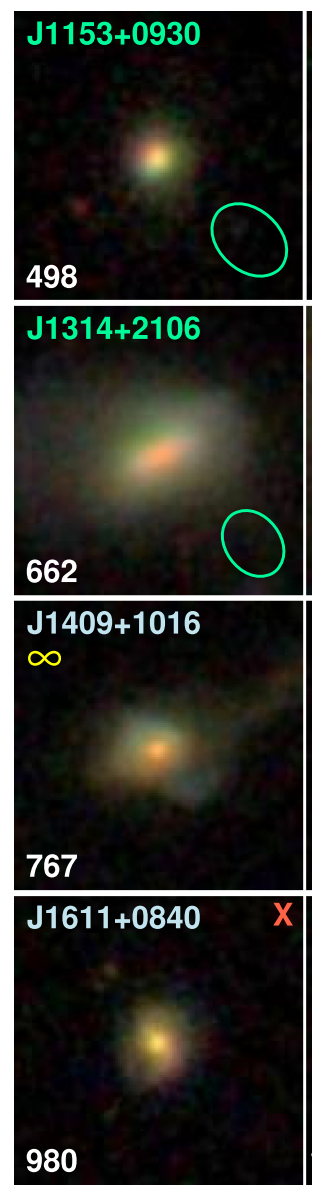
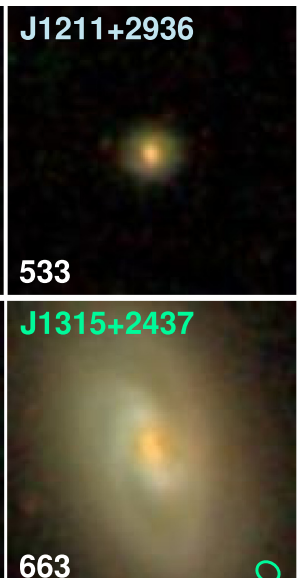

\section{$\mathrm{J} 1505+5847$}

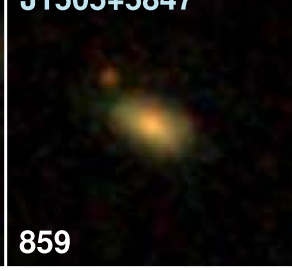

$\mathrm{J} 1645+3048$

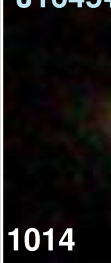

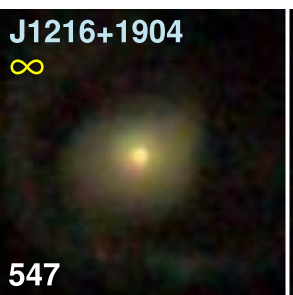
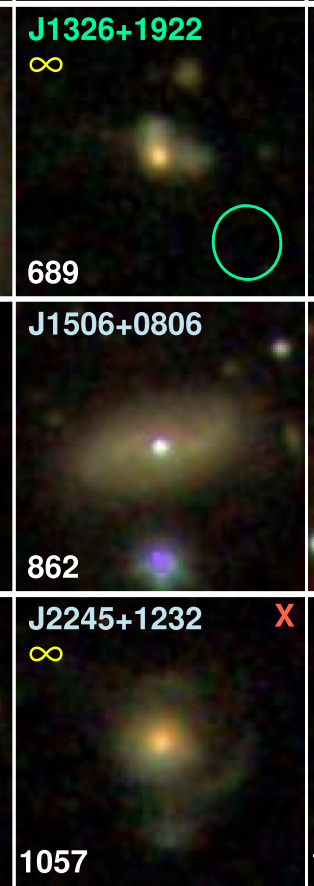

$\infty$

1057

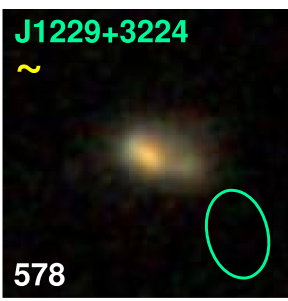

J1336+3008

J1529+0601

908

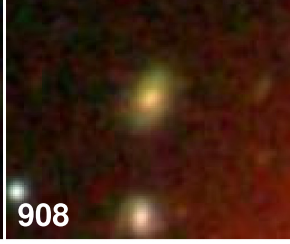

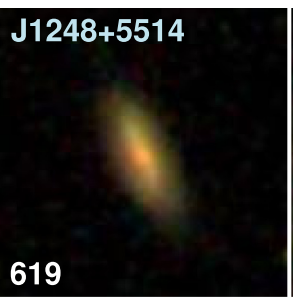

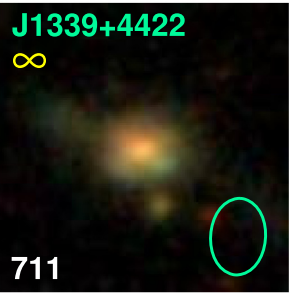

711

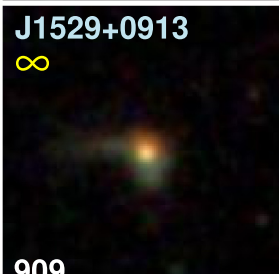

909
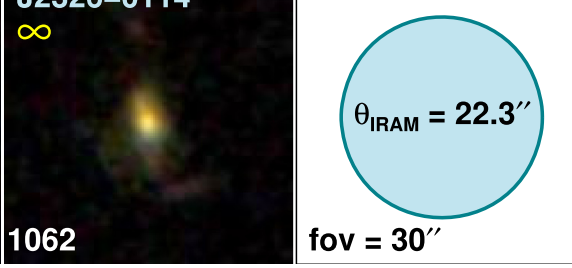

658

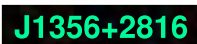

$\infty$

J1313+0207

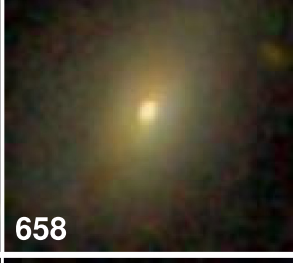

745

J1555+2955

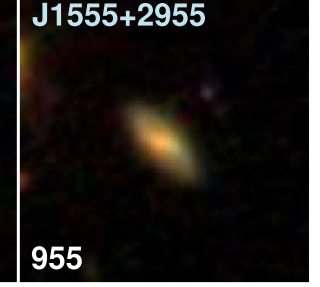

Figure 3. (Continued.)

Table 2 lists the derived properties for CARMA-observed CO-SPOGs, including the rms noise in the channel maps, the spatial extent of the molecular gas (determined by summing the total number of unmasked pixels in the moment map; see Alatalo et al. 2013 for details of moment map construction), channel widths, and total on-source hours. Derived molecular gas properties are listed in Table 3. CO luminosities are derived using the equation in Solomon \& Vanden Bout (2005):

$$
L_{\mathrm{CO}}=1.20 \times 10^{-1} \frac{S_{\mathrm{CO}} \Delta v D_{\mathrm{L}}^{2}}{1+v_{\mathrm{sys}} / c} L_{\odot},
$$

where $S_{\mathrm{CO}} \Delta v$ is the $\mathrm{CO}(1-0)$ flux (in $\mathrm{Jy} \mathrm{km} \mathrm{s}^{-1}$ ), $D_{\mathrm{L}}$ is the luminosity distance (in Mpc), $v_{\text {sys }}$ is the optically defined systemic velocity (in $\mathrm{km} \mathrm{s}^{-1}$ ), and $c$ is the speed of light (in $\mathrm{km} \mathrm{s}^{-1}$ ). Converting the $\mathrm{CO}(1-0)$ luminosity into molecular gas masses requires the assumption of a $L_{\mathrm{CO}}-M_{\mathrm{H}_{2}}$ conversion factor (Bolatto et al. 2013), which is known to be dependent on the state of the molecular gas. Interacting galaxies and ultraluminous infrared galaxies (ULIRGs) are known to have $L_{\mathrm{CO}}-M_{\mathrm{H}_{2}}$ conversions that are lower than normal, star-forming galaxies by a factor of about 5 (Downes \& Solomon 1998), though Narayanan et al. (2011) have shown that there is a large scatter above and below the standard value, even within merging systems. Sandstrom et al. (2013) showed that the nuclei of normal star-forming galaxies can exhibit $\mathrm{CO}-\mathrm{to}-\mathrm{H}_{2}$ conversion up to an order of magnitude below the Milky Way value. Determining the proper $L_{\mathrm{CO}}-M_{\mathrm{H}_{2}}$ conversion is therefore essential to deriving the molecular mass of a galaxy.

We calculate $M_{\mathrm{H}_{2}}$ with the following equation:

$$
M_{\mathrm{H}_{2}}=1.05 \times 10^{4} \frac{S_{\mathrm{CO}} \Delta v D_{\mathrm{L}}^{2}}{1+v_{\mathrm{sys}} / c} M_{\odot}
$$

assuming $X_{\mathrm{CO}}=2 \times 10^{20} \mathrm{~cm}^{-2}\left(\mathrm{~K} \mathrm{~km} \mathrm{~s}^{-1}\right)^{-1}$, the mean conversion factor presented in Bolatto et al. (2013), the derived $\mathrm{H}_{2}$ masses for our CO-SPOGs range between $10^{8.4-10.6} M_{\odot}$.

\section{DISCUSSION}

\subsection{The Molecular Gas Fraction of CO-SPOGs}

Figure 5 shows the molecular gas fraction distribution for different subsets of galaxies that contain molecular gas. Normal, star-forming galaxies from the CO Legacy Database for the GALEX Arecibo SDSS Survey (COLD GASS; top; Saintonge et al. 2011) have an average molecular gas fraction $\left\langle\log \left(f_{\mathrm{mol}, \mathrm{SF}}\right)\right\rangle=-1.29$. The classical poststarbursts (middle; French et al. 2015) have $\left\langle\log \left(f_{\text {mol,PSB }}\right)\right\rangle=-1.19$, and COSPOGs (bottom) have $\left\langle\log \left(f_{\text {mol,SPOG }}\right)\right\rangle=-0.83$. SPOGs tend to have higher gas fractions than both normal galaxies and poststarbursts. The molecular gas fractions in relation to the stellar masses range from $10^{-1.56}$ to $10^{-0.34}$, with those SPOGs at the high end of the molecular gas fraction range being comparable to 

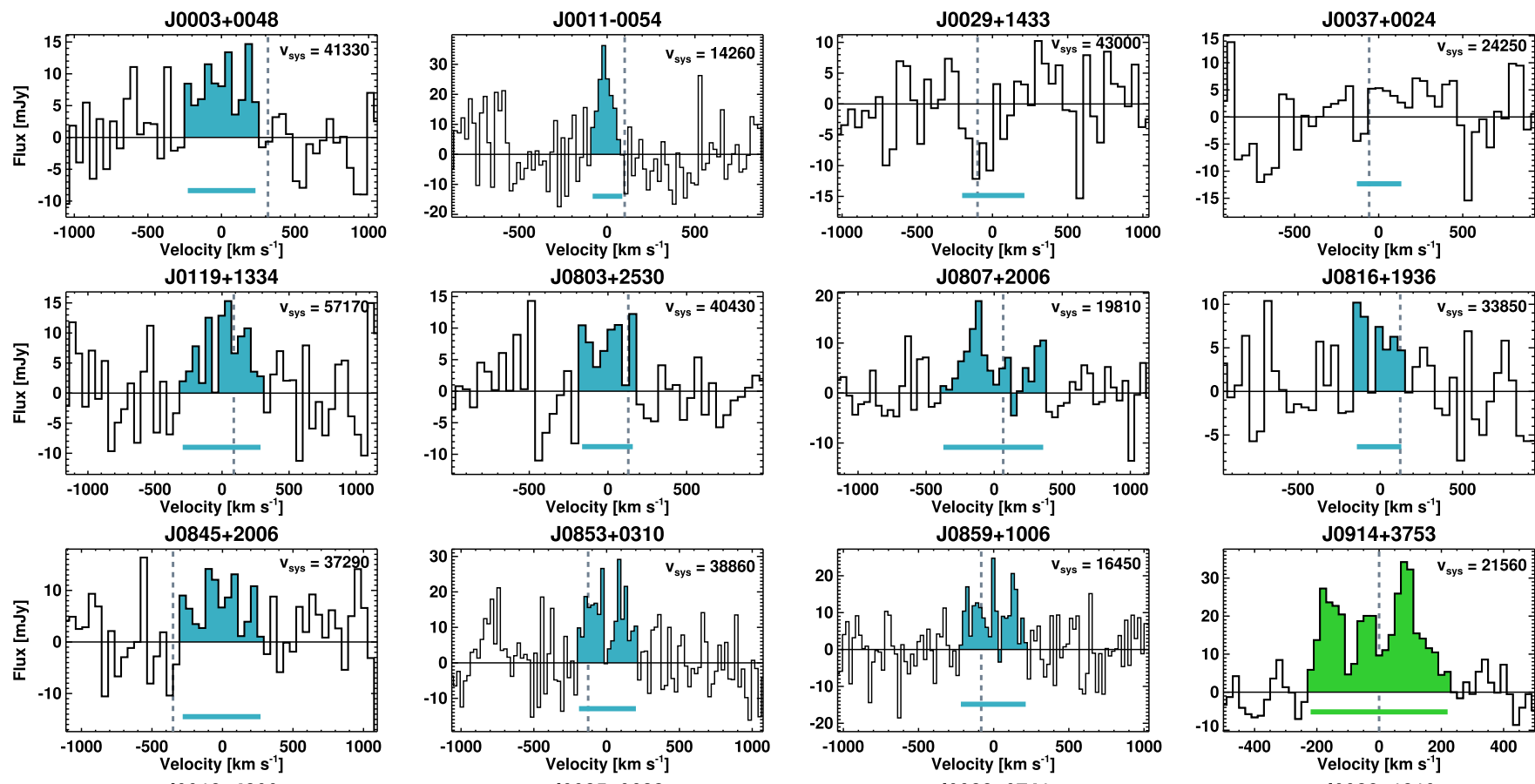

J0914+3753

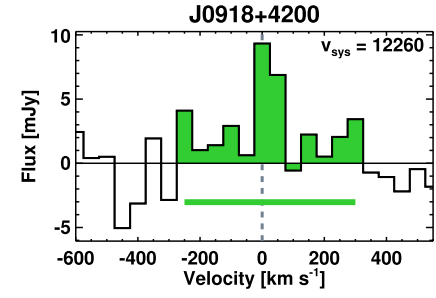

J0925+0623

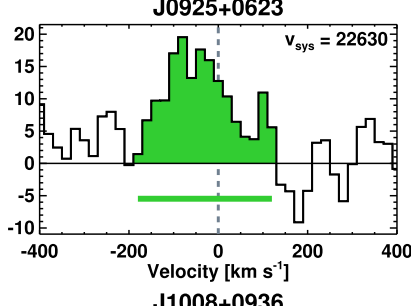

J0928+0741
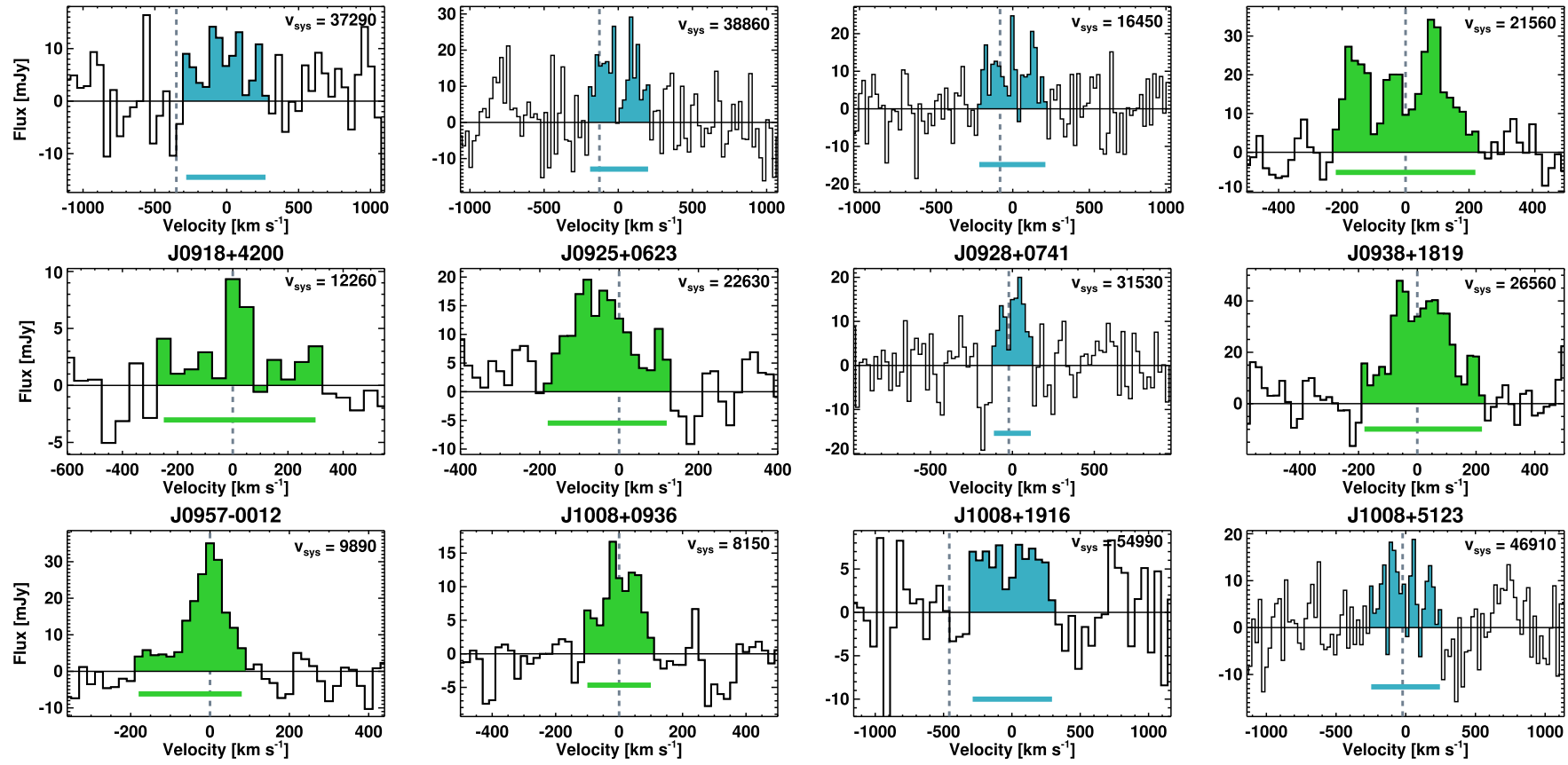

$1008+1916$
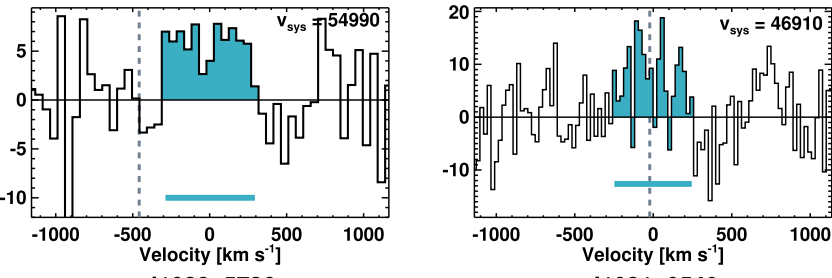

$\mathrm{J} 1026+4340$
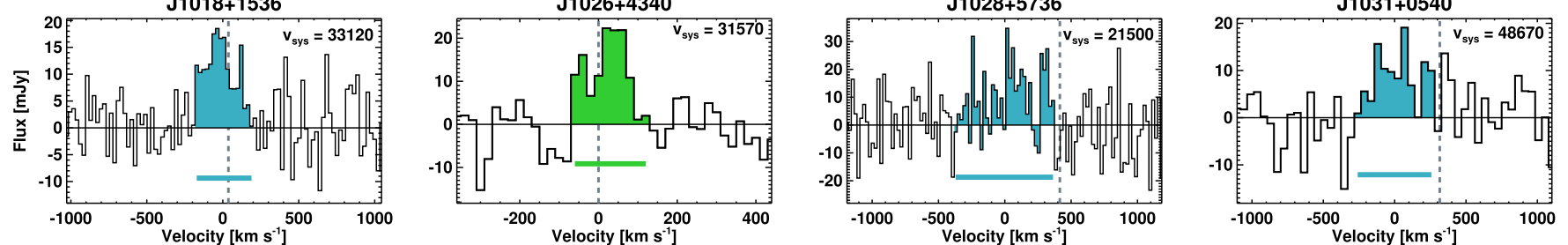

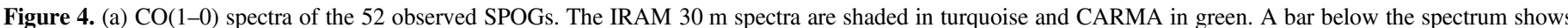

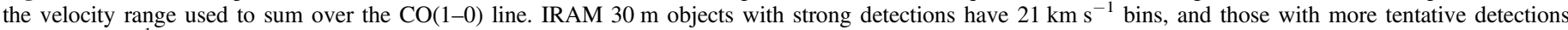

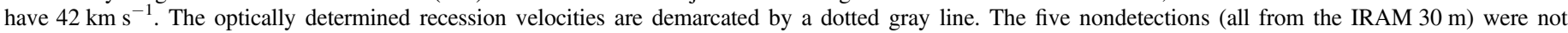

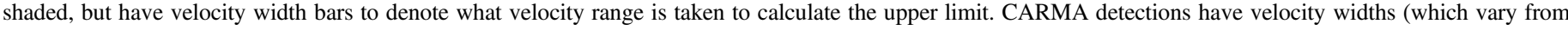

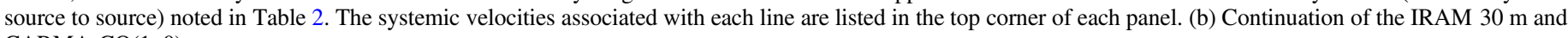
CARMA $\mathrm{CO}(1-0)$ spectra.

interactions (in which $\log \left(M_{\mathrm{H}_{2}} / M_{\mathrm{star}}+M_{\mathrm{H}_{2}}\right) \sim-0.3$; Combes et al. 2013; Kaneko et al. 2014). We ran a Mann-Whitney Utest ${ }^{13}$ (which is used in cases of small numbers) and were able to show that $f_{\text {mol }}$ in SPOGs is distinct from both star-forming galaxies $(p \approx 0)$ and poststarbursts $(p=0.00189)$, where $p$ is the probability of a null hypothesis.

13 IDL routine: rs_test.
The fraction of our CO-SPOGs classified as having disrupted morphologies is large $(37 \%-46 \% \pm 7 \%)$. This optical disruption suggests that the molecular gas should also be disrupted to some extent. Downes \& Solomon (1998) showed that in many interacting systems, the molecular gas mass predicted from the $\mathrm{CO}(1-0)$ flux was larger than the dynamical mass of the system, usually by about a factor of 5, though this is quite uncertain (Yao et al. 2003; Bolatto et al. 2013). They conclude that this is due to the nature of the molecular gas in interacting 

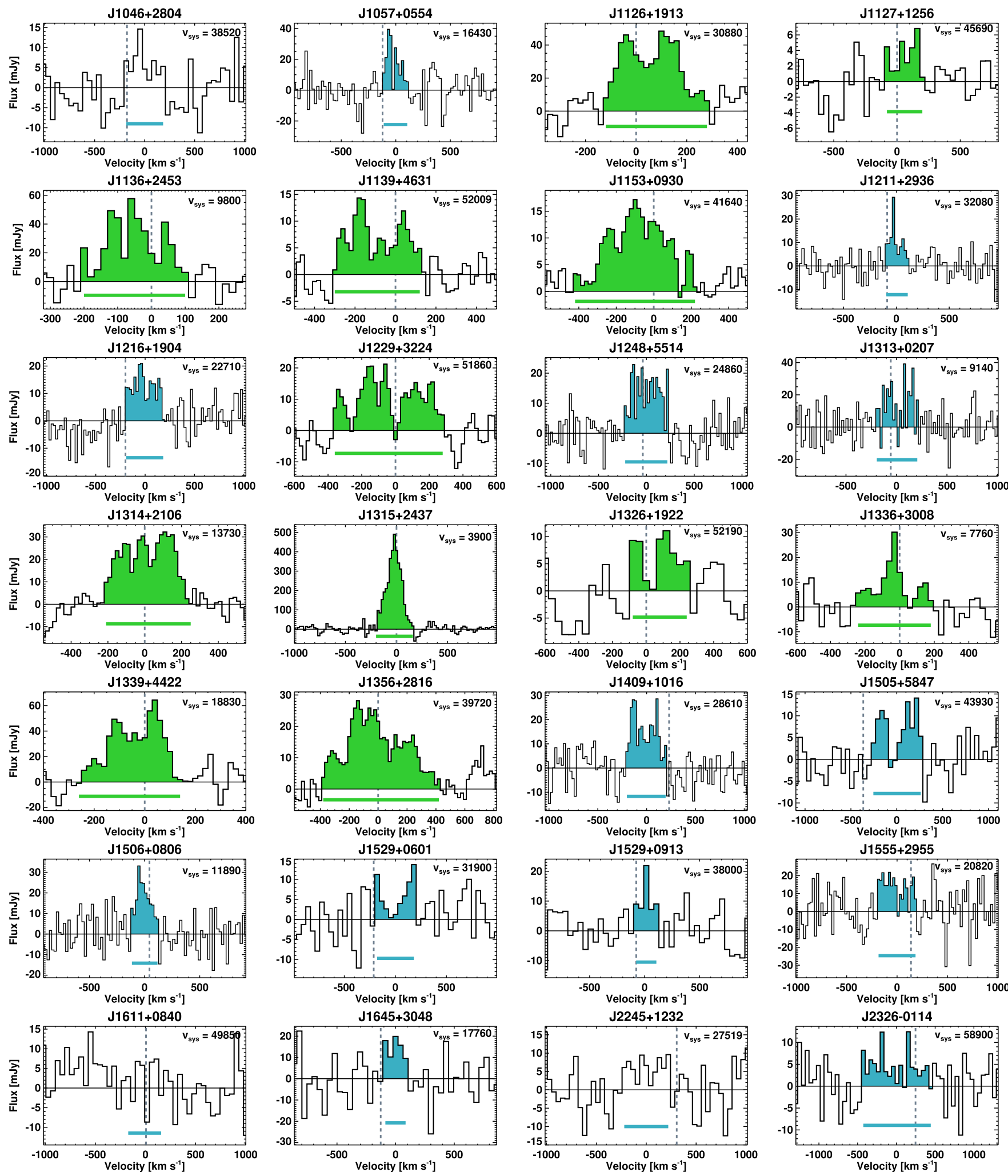

Figure 4. (Continued.)

systems being more diffuse and warm (Aalto et al. 1995; Rangwala et al. 2011), creating a more continuous coverage of CO-emitting gas rather than distributed in discrete giant molecular clouds (which would also span a smaller velocity range). If the molecular gas in SPOGs is indeed disrupted and therefore not confined to a disk as in the Milky Way, we could be overestimating the molecular gas mass by $\sim 5$. If CO-SPOGs are found on the extreme end of the conversions found by Sandstrom et al. (2013) for nuclear regions of star-forming galaxies, then the conversion could be off by as much as a 
Table 2

CARMA Observational Parameters

\begin{tabular}{|c|c|c|c|c|c|c|c|}
\hline & $\begin{array}{l}\text { SPOG } \\
\text { Name } \\
(1)\end{array}$ & $\begin{array}{l}\text { Total } \\
\text { Hrs } \\
(2)\end{array}$ & $\begin{array}{c}\text { Gain } \\
\text { Calibrator } \\
\text { (3) }\end{array}$ & $\begin{array}{c}\theta_{\text {maj }} \times \theta_{\min } \\
(\operatorname{arcsec}) \\
(4)\end{array}$ & $\begin{array}{c}\Delta v \\
\left(\mathrm{~km} \mathrm{~s}^{-1}\right)\end{array}$ & $\begin{array}{c}\mathrm{rms} \\
\left(\mathrm{mJy} \mathrm{bm}^{-1}\right) \\
(6)\end{array}$ & $\begin{array}{c}\text { Area } \\
\left(\square^{\prime \prime}\right) \\
(7)\end{array}$ \\
\hline 186 & $\mathrm{~J} 0914+3753$ & 2.98 & $0927+390$ & $10.1 \times 6.3$ & 20 & 1.4 & 222 \\
\hline 191 & $\mathrm{~J} 0918+4200$ & 5.44 & $0927+390$ & $5.5 \times 4.4$ & 50 & 1.0 & 19 \\
\hline 200 & $\mathrm{~J} 0925+0623$ & 3.16 & $0825+031$ & $8.4 \times 6.9$ & 20 & 1.4 & 142 \\
\hline 224 & $\mathrm{~J} 0938+1819$ & 2.11 & $0956+252$ & $7.7 \times 7.0$ & 20 & 1.9 & 324 \\
\hline 253 & J0957-0012 & 3.35 & $1058+015$ & $8.7 \times 7.4$ & 20 & 1.3 & 125 \\
\hline 267 & $\mathrm{~J} 1008+0936$ & 2.44 & $1058+015$ & $7.1 \times 6.6$ & 20 & 1.2 & 71 \\
\hline 305 & $\mathrm{~J} 1026+4340$ & 2.17 & $0927+390$ & $9.2 \times 6.5$ & 20 & 1.5 & 242 \\
\hline 437 & $\mathrm{~J} 1126+1913$ & 1.85 & $1159+292$ & $9.9 \times 6.4$ & 20 & 1.7 & 290 \\
\hline 439 & $\mathrm{~J} 1127+1256$ & 3.37 & $1058+015$ & $8.4 \times 7.3$ & 40 & 2.7 & 67 \\
\hline 462 & $\mathrm{~J} 1136+2453$ & 0.82 & $1224+213$ & $4.1 \times 3.5$ & 20 & 4.7 & 36 \\
\hline 470 & $\mathrm{~J} 1139+4631$ & 3.63 & $1153+495$ & $8.4 \times 6.9$ & 20 & 0.8 & 180 \\
\hline 498 & $\mathrm{~J} 1153+0930$ & 6.69 & $3 \mathrm{C} 273$ & $9.3 \times 6.8$ & 20 & 0.6 & 133 \\
\hline 578 & $\mathrm{~J} 1229+3224$ & 5.82 & $1159+292$ & $9.9 \times 6.7$ & 20 & 0.9 & 245 \\
\hline 662 & $\mathrm{~J} 1314+2106$ & 4.02 & $1310+323$ & $7.9 \times 6.1$ & 20 & 1.4 & 232 \\
\hline 663 & $\mathrm{~J} 1315+2437$ & 4.97 & $1310+323$ & $2.6 \times 2.1$ & 20 & 15.1 & 134 \\
\hline 689 & $\mathrm{~J} 1326+1922$ & 2.84 & $1310+323$ & $8.2 \times 7.5$ & 40 & 0.9 & 201 \\
\hline 704 & $\mathrm{~J} 1336+3008$ & 4.99 & $1310+323$ & $7.2 \times 5.9$ & 30 & 1.3 & 172 \\
\hline 711 & $\mathrm{~J} 1339+4422$ & 2.41 & $1419+543$ & $8.5 \times 6.1$ & 20 & 2.2 & 343 \\
\hline 745 & $\mathrm{~J} 1356+2816$ & 5.03 & $1310+323$ & $8.5 \times 6.5$ & 20 & 0.8 & 48 \\
\hline
\end{tabular}

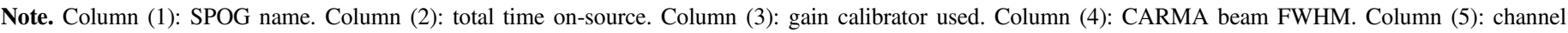
velocity width. Column (6): intensity rms per beam of CARMA images. Column (7): moment0 aperture area.

factor of 7. If all CO-SPOGs require one of these reduced conversion factors, then the molecular-to-stellar mass ratio would shift significantly, to an average ratio of $10^{-1.68}$, consistent with the COLD GASS sample (Saintonge et al. 2011), and less than the poststarburst sample (French et al. 2015).

Figure 5 also overlays the molecular gas fraction of a sample of Hickson Compact Group (HCG) galaxies, with stellar masses calculated from U. Lisenfeld et al. (2016) in preparation and $\mathrm{H}_{2}$ masses calculated from $\mathrm{CO}$ observations (Leon et al. 1998; Verdes-Montenegro et al. 1998; Martinez-Badenes et al. 2012; Lisenfeld et al. 2014), using $X_{\mathrm{CO}}=2 \times$ $10^{20} \mathrm{~cm}^{-2}\left(\mathrm{~K} \mathrm{~km} \mathrm{~s}^{-1}\right)^{-1}$. HCG galaxies span the range of $f_{\mathrm{mol}}$ values, suggestive of their large range of properties and environments (Bitsakis et al. 2010, 2011, 2014; Zucker et al. 2016). Many HCGs contain warm $\mathrm{H}_{2}$-luminous galaxies, known to host shocks (Cluver et al. 2013), which may be a better comparison sample with CO-SPOGs than interactions. Given that the SPOG criteria aimed to select objects that host shocks, the warm $\mathrm{H}_{2}$-bright galaxies serve as a reasonable comparison. Alatalo et al. (2015a) used CARMA to study the molecular gas in turbulent HCG galaxies and found that the molecular gas-to-dust ratios within these systems were consistent with the Milky Way value and predicted gas masses that were consistent with a normal CO-to- $\mathrm{H}_{2}$ conversion. Despite the fact that both types of systems host shocks, the molecular-to-stellar mass ratio for warm $\mathrm{H}_{2}$-bright $\mathrm{HCG}$ galaxies tends to be lower than that for SPOGs $\left(10^{-1.21}\right.$; Martinez-Badenes et al. 2012; Lisenfeld et al. 2014).

It is unclear which $L_{\mathrm{CO}}-M_{\mathrm{H}_{2}}$ conversion factor is more relevant to our population of CO-SPOGs. Alatalo et al. (2014a, 2016) suggested that SPOGs were at an earlier stage of quenching than other poststarburst galaxies and thus could still contain larger reservoirs of molecular gas as they undergo quenching. Observations of denser gas tracers, such as CS and $\mathrm{HCN}$, will be necessary to better infer the mass of dense gas and better define the $X_{\mathrm{CO}}$ factor.

\subsection{Comparing the $C O(1-0)$ and $22 \mu m$ Flux}

The well-known connection between mid-IR emission and star formation (Calzetti et al. 2007) is caused by hot dust reradiating UV photons from the young stars. Therefore, it is likely that a relation exists between the $22 \mu \mathrm{m}$ and $\mathrm{CO}(1-0)$ fluxes, given that the $22 \mu \mathrm{m}$ emission traces the star formation and the $\mathrm{CO}(1-0)$ traces the star-forming fuel (Rosenberg et al. 2015). The left panel of Figure 6 puts this relation to the test by comparing the $22 \mu \mathrm{m}$ and $\mathrm{CO}(1-0)$ fluxes of different samples of galaxies, including star-forming galaxies (Lisenfeld et al. 2011; Saintonge et al. 2011; Bauermeister et al. 2013), early-type galaxies (Young et al. 2011; Alatalo et al. 2013; Davis et al. 2014), radio galaxies (Evans et al. 2005), and poststarburst galaxies (French et al. 2015). The $22 \mu \mathrm{m}$ emission was obtained through a cross-matching with the ALLWISE catalog (Wright et al. 2010). In cases in which objects were flagged as extended in the Two Micron All Sky Survey (2MASS; Skrutskie et al. 2006), the extended source flux was used (w4gmag); otherwise, the profile fit flux (w4mpro) was used.

The star-forming objects of the COLD GASS survey (Saintonge et al. 2011), Evolution of molecular Gas in Normal Galaxies (Bauermeister et al. 2013), and Analysis of the interstellar Medium of Isolated GAlaxies (Lisenfeld et al. 2011) samples (black and gray dots) in Figure 6 do indeed trace a reliable relation, allowing us to predict the $\mathrm{CO}(1-0)$ flux from the $22 \mu \mathrm{m}$ flux. We used the scaling of Calzetti et al. (2007) of 
Table 3

SPOG $\mathrm{CO}(1-0)$ Values

\begin{tabular}{|c|c|c|c|c|c|c|c|c|}
\hline & $\begin{array}{l}\text { SPOG } \\
\text { Name } \\
(1)\end{array}$ & $\begin{array}{c}\text { Vel. Range } \\
\left(\mathrm{km} \mathrm{s}^{-1}\right) \\
(2)\end{array}$ & $\begin{array}{c}\mathrm{rms} \\
(\mathrm{mJy}) \\
(3)\end{array}$ & $\begin{array}{c}\mathrm{F}_{\mathrm{CO}} \\
\left(\mathrm{Jy} \mathrm{km} \mathrm{s}^{-1}\right) \\
(4)\end{array}$ & $\begin{array}{c}\mathrm{SNR}_{\mathrm{CO}} \\
(5)\end{array}$ & $\begin{array}{c}\mathrm{L}_{\mathrm{CO}} \\
\left(10^{4} L_{\odot}\right) \\
(6)\end{array}$ & $\begin{array}{c}\log \left(M_{\mathrm{H}_{2}}\right) \\
\left(M_{\odot}\right) \\
(7)\end{array}$ & $\begin{array}{r}F_{\text {gas }} \\
(8)\end{array}$ \\
\hline 1 & $\mathrm{~J} 0003+0048$ & $41070-41580$ & 7.20 & $4.19 \pm 0.78$ & 5.4 & $18.99 \pm 3.53$ & $10.22 \pm 9.5$ & 0.24 \\
\hline 4 & J0011-0054 & $14150-14350$ & 8.80 & $3.05 \pm 0.58$ & 5.3 & $1.58 \pm 0.30$ & $9.14 \pm 8.4$ & 0.06 \\
\hline 7 & $\mathrm{~J} 0029+1433$ & $42800-43200$ & 9.05 & $<7.80$ & - & $<37.58$ & $<10.52$ & 0.40 \\
\hline 13 & $\mathrm{~J} 0037+0024$ & $24100-24400$ & 7.45 & $<5.45$ & - & $<8.13$ & $<9.85$ & 0.32 \\
\hline 24 & $\mathrm{~J} 0119+1334$ & $57030-57480$ & 8.70 & $3.94 \pm 0.92$ & 4.3 & $34.41 \pm 7.99$ & $10.48 \pm 9.8$ & 0.25 \\
\hline 77 & $\mathrm{~J} 0803+2530$ & $40250-40620$ & 7.80 & $2.86 \pm 0.73$ & 3.9 & $12.28 \pm 3.11$ & $10.03 \pm 9.4$ & 0.11 \\
\hline 81 & $\mathrm{~J} 0807+2006$ & $19590-20190$ & 7.05 & $3.79 \pm 0.80$ & 4.7 & $3.79 \pm 0.80$ & $9.52 \pm 8.8$ & 0.12 \\
\hline 98 & J0816+1936 & $33670-33980$ & 6.15 & $2.01 \pm 0.52$ & 3.9 & $5.98 \pm 1.54$ & $9.72 \pm 9.1$ & 0.17 \\
\hline 142 & J0845+2006 & $36960-37530$ & 10.05 & $4.79 \pm 1.16$ & 4.1 & $16.95 \pm 4.09$ & $10.17 \pm 9.6$ & 0.27 \\
\hline 157 & $\mathrm{~J} 0853+0310$ & $38660-39080$ & 7.80 & $5.42 \pm 0.77$ & 7.1 & $21.15 \pm 2.99$ & $10.27 \pm 9.4$ & 0.20 \\
\hline 169 & J0859+1006 & $16240-16670$ & 7.65 & $4.15 \pm 0.74$ & 5.6 & $2.80 \pm 0.50$ & $9.39 \pm 8.6$ & 0.07 \\
\hline 186 & $\mathrm{~J} 0914+3753$ & $21340-21780$ & 1.40 & $7.48 \pm 0.13$ & 57.1 & $8.83 \pm 0.15$ & $9.89 \pm 8.1$ & 0.28 \\
\hline 191 & $\mathrm{~J} 0918+4200$ & $12010-12560$ & 1.04 & $1.70 \pm 0.17$ & 9.9 & $0.64 \pm 0.06$ & $8.75 \pm 7.8$ & 0.03 \\
\hline 200 & $\mathrm{~J} 0925+0623$ & $22450-22750$ & 1.42 & $3.30 \pm 0.11$ & 29.9 & $4.30 \pm 0.14$ & $9.58 \pm 8.1$ & 0.10 \\
\hline 209 & $\mathrm{~J} 0928+0741$ & $31410-31680$ & 6.20 & $2.47 \pm 0.49$ & 5.1 & $6.32 \pm 1.24$ & $9.74 \pm 9.0$ & 0.30 \\
\hline 224 & J0938+1819 & $26380-26780$ & 1.88 & $10.40 \pm 0.17$ & 61.9 & $18.78 \pm 0.30$ & $10.22 \pm 8.4$ & 0.27 \\
\hline 253 & J0957-0012 & $9710-9970$ & 1.30 & $3.75 \pm 0.09$ & 39.9 & $0.92 \pm 0.02$ & $8.90 \pm 7.3$ & 0.07 \\
\hline 267 & $\mathrm{~J} 1008+0936$ & $8050-8250$ & 1.17 & $1.88 \pm 0.07$ & 25.5 & $0.31 \pm 0.01$ & $8.43 \pm 7.0$ & 0.03 \\
\hline 268 & $\mathrm{~J} 1008+1916$ & $54750-55270$ & 6.60 & $3.21 \pm 0.74$ & 4.3 & $25.31 \pm 5.84$ & $10.35 \pm 9.7$ & 0.20 \\
\hline 270 & $\mathrm{~J} 1008+5123$ & $46650-47170$ & 6.60 & $3.84 \pm 0.73$ & 5.3 & $22.21 \pm 4.22$ & $10.29 \pm 9.6$ & 0.33 \\
\hline 293 & $\mathrm{~J} 1018+1536$ & $32920-33320$ & 5.05 & $4.12 \pm 0.48$ & 8.6 & $11.70 \pm 1.36$ & $10.01 \pm 9.1$ & 0.15 \\
\hline 305 & $\mathrm{~J} 1026+4340$ & $31509-31690$ & 1.47 & $2.51 \pm 0.09$ & 28.5 & $6.44 \pm 0.23$ & $9.75 \pm 8.3$ & 0.24 \\
\hline 308 & $\mathrm{~J} 1028+5736$ & $21120-21880$ & 10.95 & $7.32 \pm 1.40$ & 5.2 & $8.93 \pm 1.71$ & $9.89 \pm 9.2$ & 0.34 \\
\hline 322 & $\mathrm{~J} 1031+0540$ & $48490-48980$ & 9.55 & $4.66 \pm 1.02$ & 4.5 & $29.46 \pm 6.49$ & $10.41 \pm 9.8$ & 0.33 \\
\hline 349 & $\mathrm{~J} 1046+2804$ & $38300-38740$ & 8.45 & $<2.55$ & - & $<9.75$ & $<9.93$ & 0.24 \\
\hline 365 & $\mathrm{~J} 1057+0554$ & $16299-16560$ & 11.05 & $4.04 \pm 0.83$ & 4.9 & $2.70 \pm 0.56$ & $9.37 \pm 8.7$ & 0.17 \\
\hline 437 & $\mathrm{~J} 1126+1913$ & $30760-31160$ & 1.67 & $11.69 \pm 0.15$ & 78.1 & $28.69 \pm 0.37$ & $10.40 \pm 8.5$ & 0.45 \\
\hline 439 & $\mathrm{~J} 1127+1256$ & $45610-45890$ & 2.73 & $1.06 \pm 0.29$ & 3.7 & $5.81 \pm 1.58$ & $9.71 \pm 9.1$ & 0.06 \\
\hline 462 & $\mathrm{~J} 1136+2453$ & $9600-9900$ & 4.71 & $8.40 \pm 0.37$ & 23.0 & $2.01 \pm 0.09$ & $9.25 \pm 7.9$ & 0.12 \\
\hline 470 & $\mathrm{~J} 1139+4631$ & $51710-52130$ & 0.77 & $3.09 \pm 0.07$ & 43.9 & $22.10 \pm 0.50$ & $10.29 \pm 8.6$ & 0.15 \\
\hline 498 & $\mathrm{~J} 1153+0930$ & $41220-41860$ & 0.60 & $5.43 \pm 0.07$ & 80.3 & $24.61 \pm 0.31$ & $10.33 \pm 8.4$ & 0.28 \\
\hline 533 & $\mathrm{~J} 1211+2936$ & $31970-32200$ & 5.50 & $2.32 \pm 0.40$ & 5.9 & $6.12 \pm 1.04$ & $9.73 \pm 9.0$ & 0.12 \\
\hline 547 & $\mathrm{~J} 1216+1904$ & $22510-22900$ & 6.30 & $4.93 \pm 0.59$ & 8.4 & $6.35 \pm 0.75$ & $9.74 \pm 8.8$ & 0.07 \\
\hline 578 & $\mathrm{~J} 1229+3224$ & $51500-52140$ & 0.88 & $6.94 \pm 0.10$ & 69.3 & $49.38 \pm 0.71$ & $10.64 \pm 8.8$ & 0.26 \\
\hline 619 & $\mathrm{~J} 1248+5514$ & $24610-25090$ & 5.20 & $6.25 \pm 0.54$ & 11.7 & $9.82 \pm 0.84$ & $9.93 \pm 8.9$ & 0.13 \\
\hline 658 & $\mathrm{~J} 1313+0207$ & $8920-9360$ & 10.45 & $5.50 \pm 1.01$ & 5.5 & $1.13 \pm 0.21$ & $8.99 \pm 8.3$ & 0.04 \\
\hline 662 & $\mathrm{~J} 1314+2106$ & $13520-13980$ & 1.37 & $9.97 \pm 0.13$ & 75.9 & $4.71 \pm 0.06$ & $9.62 \pm 7.7$ & 0.12 \\
\hline 663 & $\mathrm{~J} 1315+2437$ & $3700-4059$ & 15.14 & $76.82 \pm 1.28$ & 59.8 & $2.88 \pm 0.05$ & $9.40 \pm 7.6$ & 0.16 \\
\hline 689 & $\mathrm{~J} 1326+1922$ & $52110-52430$ & 0.88 & $2.34 \pm 0.10$ & 23.5 & $16.89 \pm 0.72$ & $10.17 \pm 8.8$ & 0.16 \\
\hline 704 & $\mathrm{~J} 1336+3008$ & $7530-7950$ & 1.35 & $3.97 \pm 0.15$ & 26.3 & $0.59 \pm 0.02$ & $8.72 \pm 7.3$ & 0.08 \\
\hline 711 & $\mathrm{~J} 1339+4422$ & $18570-18970$ & 2.23 & $12.16 \pm 0.20$ & 60.9 & $10.90 \pm 0.18$ & $9.98 \pm 8.2$ & 0.24 \\
\hline 745 & $\mathrm{~J} 1356+2816$ & $39340-40140$ & 0.77 & $11.37 \pm 0.10$ & 117.4 & $46.77 \pm 0.40$ & $10.61 \pm 8.5$ & 0.40 \\
\hline 767 & $\mathrm{~J} 1409+1016$ & $28390-28809$ & 6.85 & $6.14 \pm 0.66$ & 9.3 & $13.11 \pm 1.41$ & $10.06 \pm 9.1$ & 0.13 \\
\hline 859 & $\mathrm{~J} 1505+5847$ & $43630-44220$ & 8.15 & $4.04 \pm 0.96$ & 4.2 & $20.06 \pm 4.77$ & $10.24 \pm 9.6$ & 0.22 \\
\hline 862 & $\mathrm{~J} 1506+0806$ & $11770-12010$ & 8.75 & $4.29 \pm 0.63$ & 6.8 & $1.53 \pm 0.22$ & $9.13 \pm 8.3$ & 0.07 \\
\hline 908 & $\mathrm{~J} 1529+0601$ & $31669-32130$ & 7.40 & $2.79 \pm 0.75$ & 3.7 & $7.22 \pm 1.94$ & $9.80 \pm 9.2$ & 0.11 \\
\hline 909 & $\mathrm{~J} 1529+0913$ & $37900-38140$ & 8.45 & $2.52 \pm 0.63$ & 4.0 & $9.42 \pm 2.34$ & $9.92 \pm 9.3$ & 0.21 \\
\hline 955 & $\mathrm{~J} 1555+2955$ & $20610-21010$ & 11.65 & $5.10 \pm 1.08$ & 4.7 & $5.68 \pm 1.20$ & $9.70 \pm 9.0$ & 0.19 \\
\hline 980 & $\mathrm{~J} 1611+0840$ & $49700-50000$ & 11.60 & $<8.75$ & - & $<57.40$ & $<10.70$ & 0.56 \\
\hline 1014 & $\mathrm{~J} 1645+3048$ & $17620-17850$ & 14.45 & $3.35 \pm 1.02$ & 3.3 & $2.63 \pm 0.80$ & $9.36 \pm 8.8$ & 0.19 \\
\hline 1057 & $\mathrm{~J} 2245+1232$ & $27400-27750$ & 9.35 & $<2.46$ & - & $<4.88$ & $<9.63$ & 0.08 \\
\hline 1062 & $\mathrm{~J} 2326-0114$ & $58470-59340$ & 8.70 & $4.51 \pm 1.27$ & 3.6 & $42.07 \pm 11.8$ & $10.57 \pm 10.0$ & 0.35 \\
\hline
\end{tabular}

Note. Column (1): SPOG name. Column (2): velocity range (optically defined, local standard of rest). Column (3): spectral rms. Column (4): CO(1-0) line flux. Column (5): the $\mathrm{S} / \mathrm{N}$ of the $\mathrm{CO}$ detections. Column (6): $\mathrm{CO}(1-0)$ luminosity. Column (7): observed mass of $\mathrm{H}_{2}$ (using the conversion factor of Bolatto et al. 2013). Column (8): molecular gas fraction, $F_{\mathrm{gas}}=\frac{M_{\mathrm{H}_{2}}}{M_{\mathrm{H}_{2}}+M_{\mathrm{star}}}$.

SFR $\propto L_{24 \mu \mathrm{m}}^{0.885}$, as well as that of Carilli \& Walter (2013) of $L_{\mathrm{FIR}} \propto L_{\mathrm{CO}}^{1.4}$ (as well as $L_{\mathrm{FIR}}$ being linearly related to the SFR; Kennicutt 1998), to derive the expected slope of the relation between $F(22 \mu \mathrm{m})$ and $S_{\mathrm{CO}} \Delta v$, setting it to $S_{\mathrm{CO}} \Delta v \propto F$ $(22 \mu \mathrm{m})^{0.632}$. Using a $4 \sigma$-clipped subset of the star-forming objects and the relation noted above, we find the following 


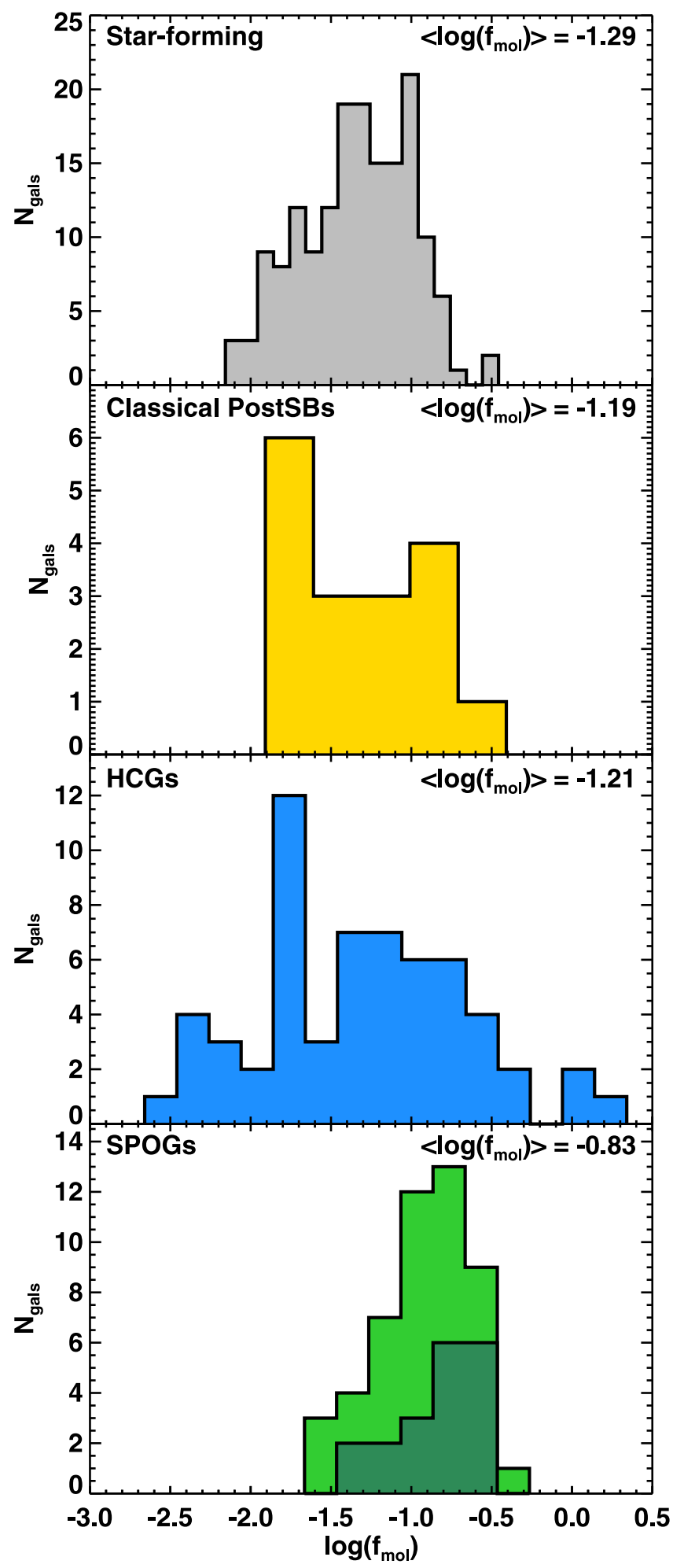

Figure 5. Distribution of molecular gas to stellar mass ratio in the COLD GASS star-forming galaxies (top; Saintonge et al. 2011), the classical poststarburst galaxies (middle, yellow; French et al. 2015), HCG galaxies middle, blue; Leon et al. 1998; Martinez-Badenes et al. 2012; Lisenfeld et al. 2014, 2016 in preparation), and CO-SPOGs (bottom, light or dark green), assuming the same $X_{\mathrm{CO}}$. The molecular-to-stellar mass fractions of CO-SPOGs are higher than those seen in both the star-forming galaxies and poststarbursts. All stellar masses were calculated as described in Section 5.1. The distribution of morphologically disrupted SPOGs (dark green) is overlaid and reflects the overall SPOG distribution faithfully. relationship between $S_{\mathrm{CO}} \Delta v$ and $F_{22 \mu \mathrm{m}}$ :

$$
\frac{S_{\mathrm{CO}} \Delta v}{\left(\mathrm{Jy} \mathrm{km} \mathrm{s}^{-1}\right)}=2.402_{-0.122}^{+0.128}\left(\frac{F_{22 \mu \mathrm{m}}}{\mathrm{mJy}}\right)^{0.623},
$$

which corresponds to the bisecting line shown in Figure 6. For regular star-forming galaxies, this relation can predict a rough $\mathrm{CO}(1-0)$ flux, though the non-star-forming samples show that this breaks down with the presence of other dominant contributors to the $22 \mu \mathrm{m}$ emission.

The early-type galaxies from the ATLAS ${ }^{3 \mathrm{D}}$ sample show a much larger scatter than the star-forming galaxies on this relation, also noted by Davis et al. (2014), with a slight mid-IR enhancement $\left(\epsilon_{\mathrm{MIR}}\right.$; defined as the ratio of the $22 \mu \mathrm{m}$ emission in the source to the expected $22 \mu \mathrm{m}$ at a given $S_{\mathrm{CO}} \Delta v$ based on the star-forming objects defined line), $\left\langle\epsilon_{\text {MIR,ETG }}\right\rangle=4.22_{-0.74}^{+0.90}$. This scatter is likely due to the fact that there are other sources of $22 \mu \mathrm{m}$ emission in early-type galaxies, including the aged stellar population and the hard ultraviolet field created by postAGB stars (responsible for the UV upturn phenomenon; O'Connell 1999; Davis et al. 2014).

A subset of the radio galaxies and quasars (Evans et al. 2005) tend to diverge the most from this relation, as their $22 \mu \mathrm{m}$ emission overpredicts the $\mathrm{CO}(1-0)$ flux with $\left\langle\epsilon_{\mathrm{MIR}, \mathrm{AGN}}\right\rangle=5.58_{-1.75}^{+2.53}$. Many of the galaxies that sit near the relation are known starbursting AGN hosts (Lanz et al. 2016). The enhancement therefore is unsurprising, given that AGNs contribute substantially to the hot dust component of a galaxy's spectral energy distribution (SED) in the mid-infrared (Ward et al. 1987; Sanders et al. 1989; Elvis et al. 1994). Thus, a quasar's divergence from this relation is due to the fact that star formation is not the primary contributor to the $22 \mu \mathrm{m}$ emission.

The poststarburst galaxies from French et al. (2015) appear to be strongly MIR-enhanced, sitting below the $S_{\mathrm{CO}} \Delta v-F_{22} \mu \mathrm{m}$ relation by a factor of $\left\langle\epsilon_{\text {MIR,PSB }}\right\rangle=10.51_{-1.60}^{+1.89}$. An additional heating mechanism (that would only heat the dust, but not increase the $\mathrm{CO}$ flux by injecting turbulence) must be present in poststarburst galaxies to cause this enhancement, such as the radiation from the intermediate-age stellar population or deeply buried AGNs.

CO-SPOGs are also a divergent population, albeit not to the extent of the poststarbursts, with all objects lying below the $S_{\mathrm{CO}} \Delta v-F_{22 \mu \mathrm{m}}$ relation set by the star-forming galaxies with $\left\langle\epsilon_{\mathrm{MIR}, \mathrm{SPOG}}\right\rangle=4.91_{-0.39}^{+0.42}$. In fact, if we limit ourselves only to strong $(\mathrm{S} / \mathrm{N} \geqslant 5)$ detections, $\left\langle\epsilon_{\mathrm{MIR}, \mathrm{SPOG}}\right\rangle$ increases to $9.75_{-1.60}^{+1.91}$. This suggests that there could be AGNs in our CO-SPOGs, but that the bolometric luminosities of these AGNs are not as strong as the extreme objects in the Evans et al. (2005) sample. The selection criteria we applied to create the CO-SPOG sample (intermediate-age stars, a lack of star formation from the line diagnostic diagram, and detectable $22 \mu \mathrm{m}$ emission) favor AGNs; thus, the $S_{\mathrm{CO}} \Delta v-F_{22 \mu \mathrm{m}}$ relation indicating the presence of AGNs is expected. How CO-SPOGs and poststarbursts compare will be presented in detail in Section 5.4.

Their positions in Figure 6 predict that SPOGs have AGN luminosities that are intermediate between the star-forming population and the quasar/radio galaxy population. Given the prevalence of intermediate-age stars found in quasars (Canalizo \& Stockton 2013), it is possible that some SPOGs in our sample may migrate to more luminous AGN phases as the galaxy transition proceeds. 


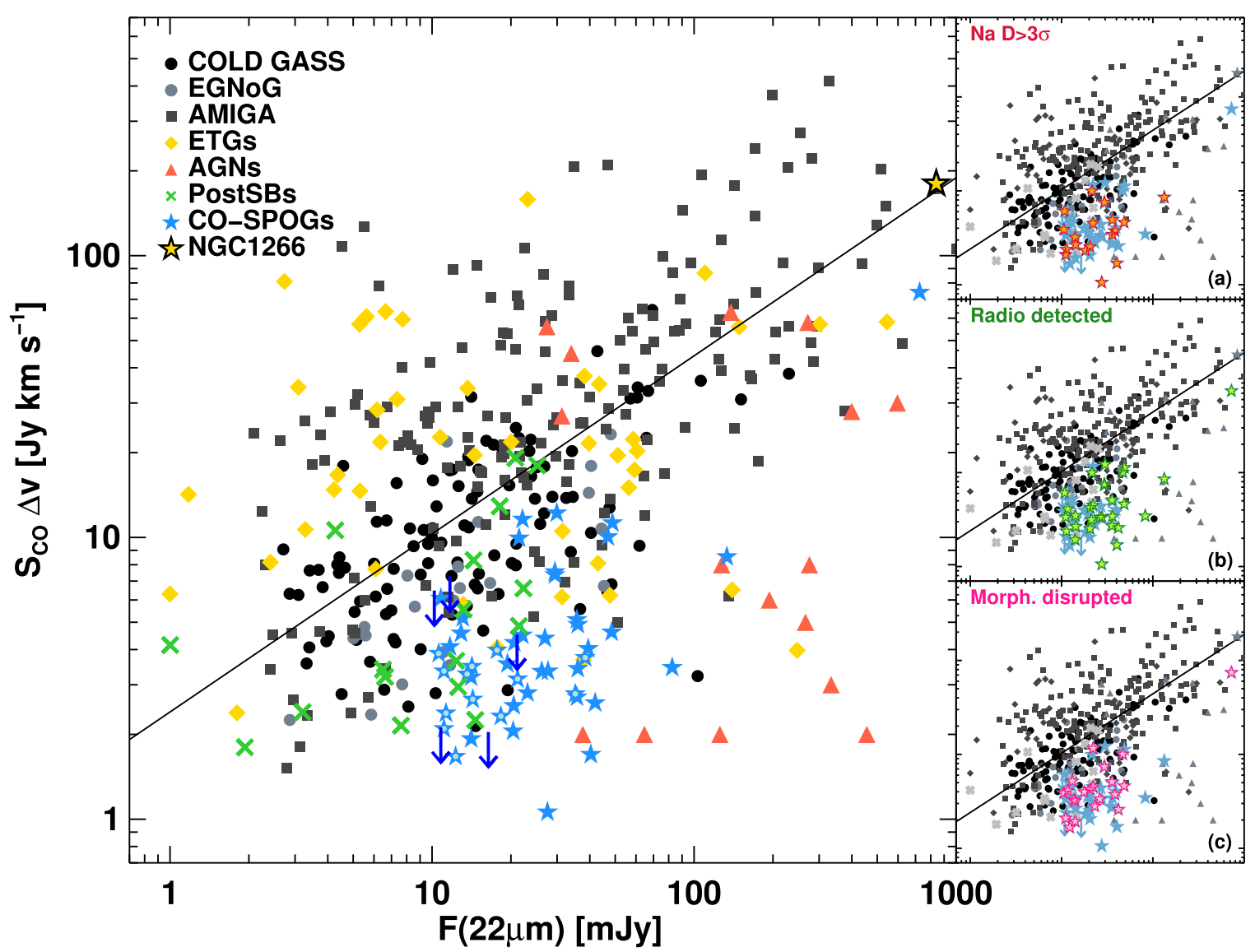

Figure 6. Left: the $22 \mu \mathrm{m}$ and $\mathrm{CO}(1-0)$ fluxes are compared for many samples, including star-forming galaxies such as COLD GASS (black dots; Saintonge et al. 2011), EGNoG (gray dots; Bauermeister et al. 2013), and AMIGA (dark gray squares; Lisenfeld et al. 2011), early-type galaxies (yellow diamonds; ATLAS ${ }^{3 \mathrm{D}}$ : Young et al. 2011; Alatalo et al. 2013; Davis et al. 2014), AGNs (red triangles; Evans et al. 2005), poststarburst galaxies (green crosses; French et al. 2015), NGC 1266 (outlined yellow star; Alatalo et al. 2011, 2014b, 2015c), and CO-SPOGs (blue stars). Light blue dots inside of the stars denote CO-SPOGs that have detections with $\mathrm{S} / \mathrm{N}$ between 3 and 5 . The $22 \mu \mathrm{m}$ and $\mathrm{CO}(1-0)$ fluxes of star-forming galaxies are well correlated (shown as a black line), with AGNs showing the strongest divergence from the relation. CO-SPOGs sit below the relation as well, though not as extremely as the AGNs. Right: the $22 \mu \mathrm{m}$ vs. CO(1-0) fluxes are shown, emphasizing the SPOGs with particular properties (symbols for other samples from the left panel have been changed to grayscale). This includes those galaxies with $3 \sigma$ excess Na I D absorption ((a); red), galaxies that were detected with FIRST ((b); green), and galaxies that were classified as clearly or possibly morphologically disrupted ((c); pink). Although radio-detected CO-SPOGs account for most of the objects that fall the farthest from the $F_{22}-F_{\mathrm{CO}}$ relation, they do not universally exhibit quasar-like $22 \mu \mathrm{m}$ excess relative to their CO fluxes.

The right panels of Figure 6 have taken the relation and broken CO-SPOGs down into subpopulations based on their NaID properties (top), radio detections (middle), and morphological properties (bottom) to determine whether any of these properties influence $\epsilon_{\mathrm{MIR}}$. Our CO-SPOGs have $\left\langle\epsilon_{\mathrm{MIR}, \mathrm{SPOG}}\right\rangle=4.91_{-0.39}^{+0.42}$, falling off the star-forming relation by a factor of $\approx 5$. Objects with either radio emission or an $\mathrm{NaID}$ enhancement (detailed in Section 5.5) appear to fall slightly farther from the relation with $\left\langle\epsilon_{\mathrm{MIR}, \text { radio }}\right\rangle=4.95_{-0.55}^{+0.62}$ and $\left\langle\epsilon_{\mathrm{MIR}, \mathrm{Na} \mathrm{ID}}\right\rangle=5.40_{-0.73}^{+0.85}$, respectively, but agree within the uncertainty. CO-SPOGs that were classified as morphologically disrupted were found to have smaller deviations from the relation, $\left\langle\epsilon_{\mathrm{MIR}, \text { dissupted }}\right\rangle=4.37_{-0.43}^{+0.47}$. In all of these cases, the overall $\epsilon_{\text {MIR }}$ was not sufficiently deviant from the overall CO-SPOG population to be distinct, and thus we cannot conclude whether the presence of any of these properties enhances or suppresses the mid-IR emission as compared to the $\mathrm{CO}$ flux (relative to star-forming galaxies).

\subsection{Ambiguity of Star Formation Tracers in CO-SPOGs}

In CO-SPOGs, shocks can dominate the ionized gas emission (Allen et al. 2008; Rich et al. 2011), including [O II] and $\mathrm{H} \alpha$. UV emission suffers from large uncertainties due to extinction and contamination due to non-star-formation- dominated phenomena, such as heating by intermediate-age stars. Finally, as Figure 6 laid out, the $22 \mu \mathrm{m}$ emission can be significantly contaminated by the presence of an AGN. In NGC 1266, the $22 \mu \mathrm{m}$ emission overestimates the SFR by a factor of 10 (Alatalo et al. 2011), despite star formation being suppressed by a factor of $>50$ (Alatalo et al. 2015c). While centimeter-wave radio emission is a sensitive tracer of recent star formation (Condon 1992), the continuum emission at $1.4 \mathrm{GHz}$ can also be contaminated by the synchrotron emission from an AGN (Zensus 1997; Laor \& Behar 2008).

Constructing an SED that includes far-infrared emission is by far the most reliable tracer of star formation in all systems except those with the most deeply buried AGNs, because the cold dust nearly unambiguously traces star formation originating in imbedded clouds. It is possible that a single data point on the Rayleigh-Jeans tail of the dust continuum blackbody could reduce the uncertainty of SFRs in ambiguous systems, such as SPOGs. For instance, an observation at $850 \mu \mathrm{m}$ would be able to anchor the blackbody. If we assume that the dust temperatures do not vary much away from $T_{\mathrm{d}} \approx 25 \mathrm{~K}$ (Scoville et al. 2014), we should be able to use the 22 and $850 \mu \mathrm{m}$ points to interpolate the modified blackbody for the dust continuum across, inferring a star formation rate. While this method is not 
as precise as fitting a full SED and dust continuum (with far-IR data near the peak of the blackbody), it could significantly improve SFR estimates for SPOGs.

\subsection{CO-SPOGs and CO-detected Poststarbursts}

Alatalo et al. (2016) argue that the SPOG criterion might identify objects at an earlier stage of transition than the classical poststarburst criterion. French et al. (2015) showed that $53 \%$ of poststarburst galaxies contain non-negligible reservoirs of molecular gas, modifying the standard picture of galaxy evolution in which a galaxy expels its interstellar medium before transitioning (Hopkins et al. 2006). We have also shown that compared to their molecular gas content, the poststarbursts of French et al. (2015) are significantly $22 \mu \mathrm{m}$ enhanced.

The French et al. (2015) poststarburst sample has reservoirs of molecular gas ranging from $10^{8.6}$ to $10^{9.8} M_{\odot}$ in mass, and molecular-to-stellar mass fractions between $10^{-2}$ and $10^{-0.5}$. In that sample, the majority of the poststarburst galaxies contain ionized gas line ratios that are consistent with LINERs, and a substantial fraction show disrupted optical morphologies. Of the $17 \mathrm{CO}$-detected poststarbursts from the French et al. (2015) sample, 15 were detected in the $22 \mu \mathrm{m}$ WISE band with an $\mathrm{S} / \mathrm{N}>3$; thus, all but two French et al. (2015) poststarbursts would have surpassed the $22 \mu \mathrm{m}$ criterion of the CO-SPOGs. There is no difference in average $f_{\text {mol }}$ in the poststarburst sample when we remove the two $22 \mu \mathrm{m}$ nondetected poststarbursts from consideration.

CO-SPOGs compare well to the French et al. (2015) poststarbursts in ionized gas ratios and $22 \mu \mathrm{m}$ properties, and morphologies, including both sets presenting substantial LINER-like ionized gas line diagnostics, similar detection rates in WISE $22 \mu \mathrm{m}$ emission, and a similar fraction of objects present morphological disruptions. Due to a selection against strong [O III] emission, the French et al. (2015) CO-detected poststarbursts have an average EW[O III] that is an order of magnitude smaller $\left(\left\langle\mathrm{EW}[\mathrm{O} \mathrm{III}]_{\mathrm{PSB}}\right\rangle=0.60\right)$ than what is found for CO-SPOGs $\left(\left\langle\mathrm{EW}[\mathrm{O} \mathrm{III}]_{\mathrm{COSPOG}}\right\rangle=5.74\right)$. The poststarbursts of French et al. (2015) show low CO fluxes compared to their $22 \mu \mathrm{m}$ fluxes, and CO-SPOGs seem to contain larger reservoirs of molecular gas (Figure 7; assuming equivalent values for $\left.X_{\mathrm{CO}}\right)$. We also see discrepancies between SPOGs and poststarbursts in the molecular-to-stellar mass ratio (Figure 5), where SPOGs exhibit much higher gas fractions. ${ }^{14}$

The fact that SPOGs contain more molecular gas than French et al. (2015) poststarbursts could suggest one of the following. Either (1) the SPOG criteria allowed for galaxies that contained more substantial reservoirs of molecular gas in the first place, due to not strictly excluding $\mathrm{H} \alpha$ or [O II] emission, or (2) SPOGs are in an earlier phase of their transition; possibly both of these effects are at play. It is also possible that in some $\mathrm{CO}$ SPOGs, the bulk of star formation is taking place behind an optically thick screen, although IFU studies of objects at different merger stages have shown that such "skin effect" phenomena are rare (Rich et al. 2015) and do not explain the excess $22 \mu \mathrm{m}$ enhancement present in the poststarbursts. Optical IFU studies of CO-SPOGs would help elucidate whether this effect is present in any of our objects.

\footnotetext{
14 A standard $X_{\mathrm{CO}}$ was used both for poststarbursts and for CO-SPOGs, since the time differentiation between the SPOG phase and poststarburst phase is sufficiently short that we do not expect a dramatic change in the conversion outside of the scatter seen in different merger simulations (Narayanan et al. 2011).
}

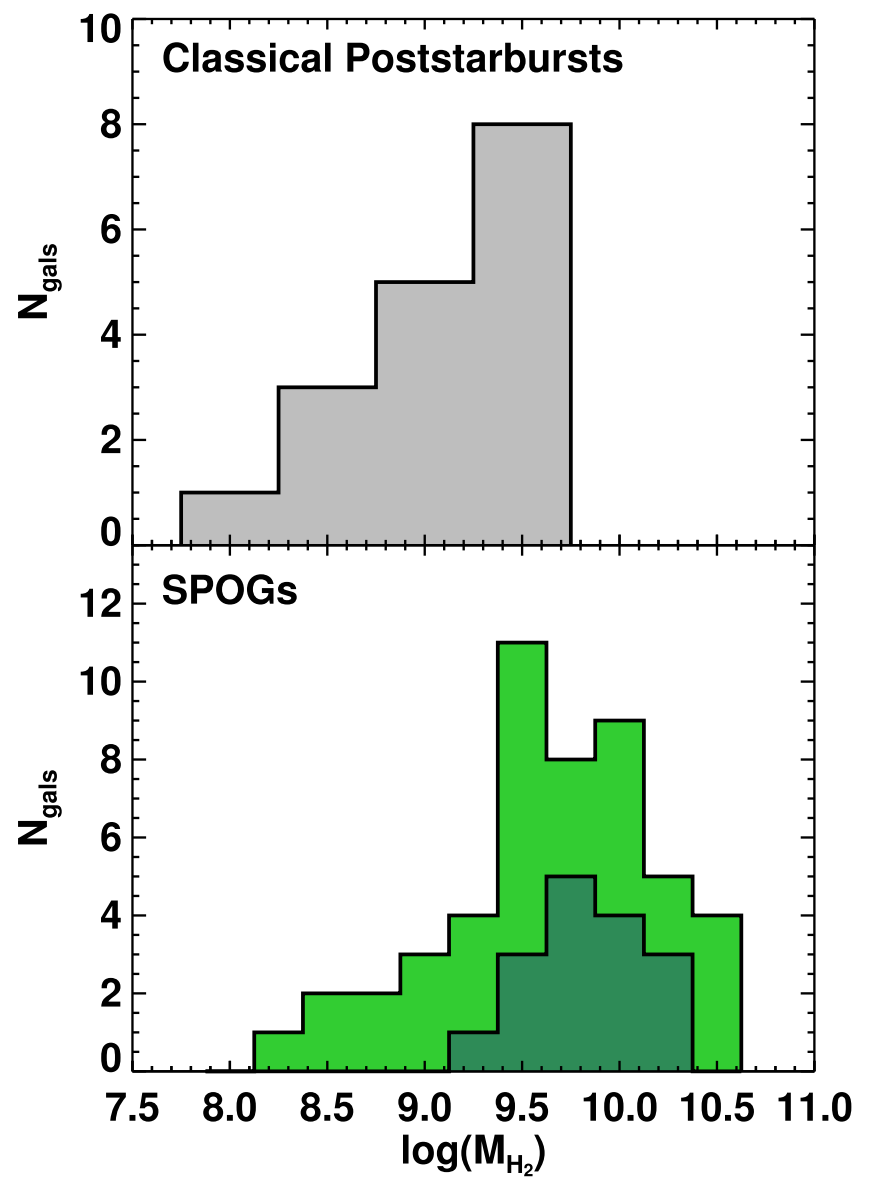

Figure 7. Distribution of molecular gas in the classical poststarburst galaxies (top; French et al. 2015) and CO-SPOGs (bottom, light green). The distribution for the morphologically disrupted SPOGs is overlaid in dark green and reflects the overall SPOG distribution faithfully. SPOGs have significantly more molecular mass than poststarbursts.

Figure 9 in Alatalo et al. (2016) indicates that SPOGs are bluer than poststarburst galaxies (Goto 2007; Yesuf et al. 2014; Rowlands et al. 2015) and lie on the blueward side of the green valley, and Figure 2 confirms that CO-SPOGs follow the colors of the general SPOG population. SPOG optical colors may be bluer than poststarbursts because they have truncated their star formation more recently and thus have a larger population of blue stars (to contribute to the galaxy colors). A large-scale stellar population analysis of these two populations would be able to better pinpoint how SPOGs and poststarbursts are related, but the larger reservoir of molecular gas in SPOGs could suggest that they are at an earlier phase of evolution, if depletion of the molecular reservoir is related to the time since the start of a galaxy's transition.

If galaxy transition does correlate with the depletion of molecular material, then the larger gas reservoirs and gas fractions are suggestive of a timescale effect, which correlates with the phase of transition. Alatalo et al. (2015a) used interferometric $\mathrm{CO}$ measurements to show that in some warm $\mathrm{H}_{2}$-bright $\mathrm{HCG}$ galaxies, the expulsion of the molecular reservoir was not required for a galaxy to transform. Instead, these authors argue that the inhibition of the molecular gas to form stars was a more important factor in predicting a galaxy's color. Whether CO-SPOGs or the poststarbursts of French et al. (2015) show this impact too will require sufficiently resolved molecular gas and accurate star formation rates to study 


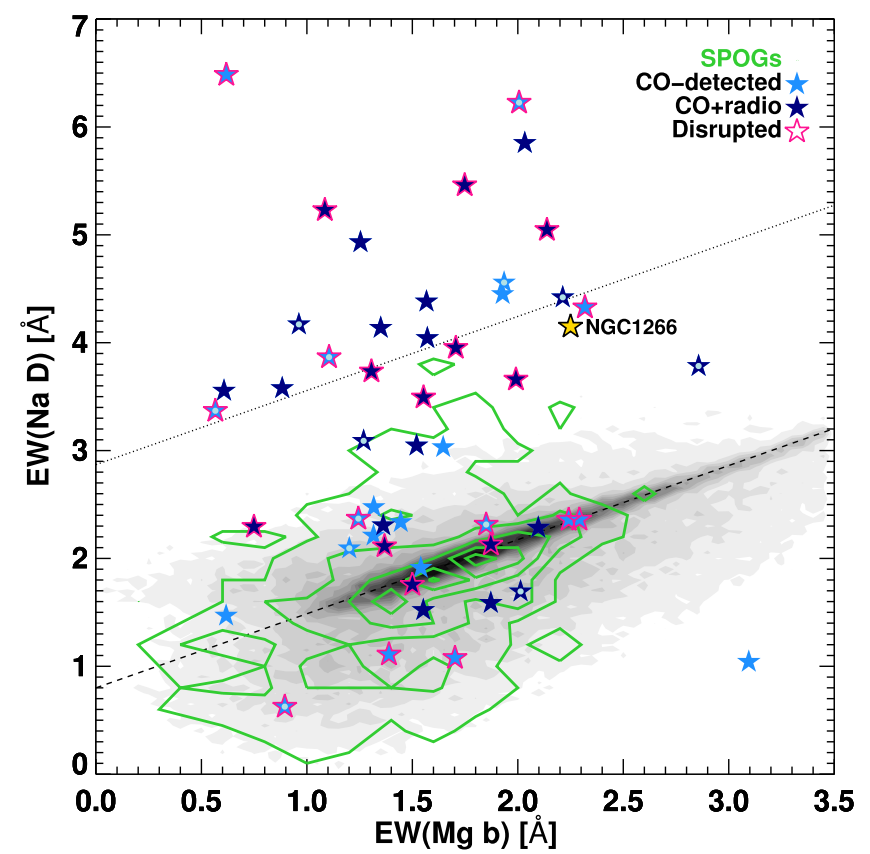

Figure 8. Equivalent widths $\mathrm{EW}(\mathrm{Mg} \mathrm{b})$ vs. EW(Na I D) of the ELG sample (grayscale; Alatalo et al. 2016) compared with the entire SPOGS sample (green contours). The dashed line represents the empirical relation found in Alatalo et al. (2016). The dotted line represents a $3 \sigma$ departure from the empirical relation. The CO-SPOG sample is shown (stars), including FIRST radio detections (dark blue) and nondetections (light blue), as well as NGC 1266 (gold; Davis et al. 2012). CO-SPOGs with a light blue dot in their centers are detected with $\mathrm{S} / \mathrm{N}$ between 3-5. SPOGs have $\mathrm{Na}$ I D compared to $\mathrm{Mg}$ b that is enhanced beyond what is seen in normal star-forming galaxies in the ELG sample. The $\mathrm{CO}(1-0)$ detected objects show even more enhanced $\mathrm{Na}$ I D characteristics compared to non-CO-observed SPOGs, with 19 (37\%) objects beyond the $3 \sigma$ boundary defined by the normal (ELG-defined) relation. There does not appear to be a difference between the objects observed by CARMA and those observed by the IRAM $30 \mathrm{~m}$. Clearly and possibly morphologically disrupted SPOGs are outlined in pink. Radio-detected SPOGs tend to have the largest Na I D excess, and radio nondetected, nondisrupted SPOGs have the least excess. Most radio nondetected SPOGs that do show Na I D excess at the $>3 \sigma$ level also show morphological disruptions.

whether a star formation suppression phase globally manifests in transforming galaxies or whether it is a special phenomenon seen in the compact group environment.

\subsection{Na I D In CO-SPOGs: Do They Contain Neutral Winds?}

Alatalo et al. (2016) showed that, as a population, SPOGs have a higher fraction of sources with $\mathrm{NaID}$ properties that require an interstellar component compared to the (starformation-dominated) ELG sample. Figure 8 shows the underlying distribution from the ELG sample (in grayscale), along with the SPOG distribution (green contours), where we see that there is an $\mathrm{Na}$ I D-enhanced wing. Overplotted are COSPOGs (stars), labeled based on whether they were detected at 1.4 GHz by FIRST (dark blue) or not (light blue), and whether they are disrupted (magenta outline). We can see that the COSPOGs are even more NaID enhanced than the underlying SPOG population. We ran the Mann-Whitney U-test to test whether the $\mathrm{Na}$ I D properties of CO-SPOGs could be randomly drawn from the SPOG sample, and found that the null hypothesis was ruled out $(p \approx 0)$. A total of $19(37 \% \pm 7 \%)$ of the CO-SPOGs contain $\mathrm{NaID}$ emission above the $3 \sigma$ boundary defined by the $\mathrm{NaID}-\mathrm{Mg}$ b relation of the ELG sample (Equation (10) in Alatalo et al. 2016). This excess suggests the possibility that many CO-SPOGs host interstellar
Table 4

Na I D Properties of SPOGs

\begin{tabular}{lrccc}
\hline \hline Type & \multicolumn{1}{c}{$N_{\text {obj }}$} & $\begin{array}{c}\epsilon_{\mathrm{NaD}} \\
(1)\end{array}$ & $\begin{array}{c}\epsilon_{\mathrm{NaD}, 10} \\
(4)\end{array}$ & $\begin{array}{c}\epsilon_{\mathrm{NaD}, 90} \\
(5)\end{array}$ \\
\hline ELG & 159,387 & -0.073 & -1.142 & 0.833 \\
ELG 22 $\mu \mathrm{m}>3 \sigma$ & 71,301 & -0.009 & -0.966 & 1.023 \\
SPOGs & 1,067 & -0.043 & -1.111 & 1.656 \\
SPOG 22 $\mu \mathrm{m}>3 \sigma$ & 491 & 0.115 & -0.856 & 2.105 \\
CO-SPOGs & 52 & 1.421 & -0.485 & 3.273 \\
CO-SPOGs, S/N $>5$ & 34 & 1.452 & -0.341 & 3.463 \\
CO-SPOGs, radio & 30 & 1.984 & -0.072 & 3.463 \\
CO-SPOGs, morph. & 24 & 1.495 & -0.641 & 3.686 \\
\hline
\end{tabular}

Note. Column (1): galaxy type. Column (2): total number of objects in each class. Column (3): median Na I D enhancement of each sample. Column (4): the 10th percentile Na I D enhancement. Column (5): the 90th percentile Na I D enhancement.

winds (Rupke et al. 2005; Veilleux et al. 2005; Murray et al. 2007; Park et al. 2015).

Figure 8 indicates that a fraction of CO-SPOGs host Na I D properties as extreme as what is seen in AGN-driven outflow hosts (Rupke \& Veilleux 2011, 2015), including NGC 1266 (Alatalo et al. 2011; Davis et al. 2012; Nyland et al. 2013). The extreme $\mathrm{Na} I \mathrm{D}$ widths of these sources suggest that they may host an AGN-driven molecular outflow like NGC 1266. If so, it would indeed shed light on how such outflows relate to galaxy transformation. However, it is also possible that NaID enhancements are due to unsettled neutral gas along the line of sight instead.

We have investigated how Na I D absorption changes across the ELG sample. Table 4 shows the median values and 10th and 90th percentile values of the ELG, SPOG, and CO-SPOG subsamples. ${ }^{15}$ Overall, objects that have been detected in the $22 \mu \mathrm{m}$ band of WISE appear to show more enhanced Na I D in both the ELG and the SPOG samples. In the case of galaxies within the ELG (and thus star-formation-dominant) sample, this $\mathrm{NaID}$ enhancement might be able to be explained by neutral winds being launched by the starburst (Murray et al. 2007; Park et al. 2015; Sarzi et al. 2016), which would correlate with the $22 \mu \mathrm{m}$ hot dust emission (and therefore the star formation rate). The SPOG selection criteria eliminated strong star-formers (based on ionized gas emission-line ratios; Baldwin et al. 1981; Veilleux \& Osterbrock 1987; Kewley et al. 2006), so the $\mathrm{NaID}$ excess and $22 \mu \mathrm{m}$ emission are possibly from an alternative source.

As discussed in Section 2, the ramifications of this selection are that our CO-SPOGs likely favor the presence of AGNs. SPOGs also follow the trend that objects hosting radio emission and $22 \mu \mathrm{m}$ emission show a significant Na ID enhancement when compared to other samples, including radio or $22 \mu \mathrm{m}$ nondetected SPOGs. Radio-detected SPOGs exhibit a median NaID enhancement (defined as the deviation from the mean relation of the ELG of $\mathrm{NaID}=(0.685 \mathrm{Mgb}+0.8)$ from Alatalo et al. 2016) of $\left\langle\epsilon_{\mathrm{NaD} \text {,radio }}\right\rangle=1.421$, and $13 / 19(68 \% \pm 11 \%)$ objects found with $3 \sigma \mathrm{NaID}$ enhancements were radio detected (although $57 \% \pm 7 \%$ of the CO-SPOGs have been detected in radio). Objects that were not detected in FIRST have a median $\mathrm{Na}$ I D enhancement of $\left\langle\epsilon_{\mathrm{NaD} \text {,non-radio }}\right\rangle=0.547$. CO-SPOGs with radio emission have higher $\mathrm{Na} I \mathrm{D}$ enhancements than objects that

\footnotetext{
15 It is of note that there is very little difference in the $\mathrm{Na}$ I D properties of strong $(\mathrm{S} / \mathrm{N} \geqslant 5)$ detections and tentative $(3 \leqslant \mathrm{~S} / \mathrm{N}<5)$ detections.
} 
do not. This is further supported by the results of the MannWhitney U-test, which was able to rule out that the NaID properties of radio nondetected CO-SPOGs were drawn from the same distribution of radio-detected CO-SPOGs with the probability of a null hypothesis of $p<0.04$. Given that AGN activity is a probable origin of the radio emission, we suggest that AGNhosting SPOGs are the most likely to contain enhanced Na ID.

We also tested whether the morphologies of the SPOGs had a strong influence on the $\mathrm{Na}$ I D enhancement, given that many objects that host strong neutral winds are starbursts in ULIRGs, which are mostly major mergers (Veilleux et al. 2005). The overall effect of morphological disruption seems to have a slight impact on the NaID enhancement, with median $\left\langle\epsilon_{\mathrm{NaD} \text {, disrupted }}\right\rangle=1.495$, slightly larger than the median for galaxies that were not classified as being disrupted of $\left\langle\epsilon_{\mathrm{NaD}}\right.$, undisturbed $\rangle=1.206$. Therefore, morphological disruption appears to have a smaller influence on the $\mathrm{Na}$ I D enhancement of CO-SPOGs than the presence of radio emission.

Sarzi et al. (2016) showed that in a sample of 456 nearby galaxies, sources with $\mathrm{NaID}$ enhancements attributable to interstellar winds (as opposed to $[\mathrm{Na} / \mathrm{Fe}]$ overabundances as is seen in the most massive galaxies; Jeong et al. 2013) were not observed in objects detected with very long baseline interferometry (Deller \& Middelberg 2014). These authors concluded that the majority of $\mathrm{Na}$ I D-enhanced objects were prolifically star-forming galaxies with neutral winds that were star formation driven, and that $\mathrm{NaID}$ winds originate most commonly in star-forming galaxies due to star formation driving. In CO-SPOGs, it appears that there are (radio-detected) AGNs and $\mathrm{Na}$ I D enhancements in the same objects, consistent with the results of Lehnert et al. (2011) that 33\% of radio galaxies within SDSS also contain Na I D enhancements. The lack of this type of object within the Sarzi et al. (2016) sample suggests that they could be quite rare or are possibly a short and violent phase in galaxy evolution, in which the star formation has quenched and an AGN is driving a neutral wind that is stirring up the remaining interstellar medium. The SPOG criterion selected against star-forming objects (which is able to rule out strong starbursts that are needed to drive winds; Alatalo et al. 2016) and thus includes many more galaxies with LINER emission, as opposed to the Sarzi et al. (2016) sample.

Further studies are needed, including observations with higher spectral resolution to investigate the shape and structure of the Na I D line, to look for outflows (Rupke et al. 2005; Veilleux et al. 2005; Rupke \& Veilleux 2011). Integral field spectrographs will be able to determine the extent of the $\mathrm{Na}$ ID absorption, provide high-resolution kinematics to determine the neutral mass flux, and compare the Na ID kinematics to the stellar kinematics. Deeper $\mathrm{CO}$ observations might detect broad molecular wings, resulting in the measurement of a range of the mass outflow (and mass escape) rates observed in transitioning objects. In-depth studies of these objects' star formation rates and molecular gas distributions will shed light on how often star formation is suppressed, leading to conserving and extending the lifetime of the molecular gas as galaxies undergo transformations from late types to early types. Deep X-ray observations will put limits on the AGN luminosity, helping to determine the range in energy budgets these systems might exhibit, and two-dimensional stellar population studies might be able to provide a range of timescales over which triggering mechanisms started the process of star formation quenching in these systems.

\section{SUMMARY}

We have followed up 52 of the WISE $22 \mu$ m-detected objects from the Shocked POststarburst Galaxy Survey using the IRAM $30 \mathrm{~m}$ and CARMA to search for $\mathrm{CO}(1-0)$. We were able to detect 47 of these 52 SPOGs to at least $3 \sigma$ significance.

1. The requirement of detected $22 \mu \mathrm{m}$ emission, combined with ionized gas emission-line ratios inconsistent with star formation, likely biases our CO-SPOG sample toward the detection of AGNs. Despite this, a large subset of our objects do not have line ratios consistent with a pure Seyfert nucleus.

2. The CO-SPOG sample appears to span the color phase space of the SPOG parent sample, though with a bias toward the more massive SPOGs. A morphological investigation was undertaken to visually classify whether an object was disrupted, finding that $37 \%-46 \% \pm 7 \%$ of our CO-SPOG sample shows signs of morphological disruptions.

3. The molecular gas fractions exhibited by the CO-SPOGs are larger than those in normal star-forming galaxies and those in a sample of traditionally identified poststarburst galaxies, most of which were detected in $22 \mu \mathrm{m}$ emission. The molecular gas fractions identified in our sample are consistent with those seen in interactions, supported by our identification of a large fraction of morphologically disrupted objects, although it is possible that our $22 \mu \mathrm{m}$ selection has biased the sample to select objects with buried ongoing star formation, which will require further observations to measure accurately.

4. We used star-forming galaxies to derive a relation between the $22 \mu \mathrm{m}$ flux from WISE and the $\mathrm{CO}(1-0)$ flux, finding that they were in agreement (supporting the claim that the mid-IR in star-forming galaxies is originating from the star formation itself) and that quasars and radio galaxies fall off this relation. SPOGs in general sit between star-forming galaxies and quasars/radio galaxies, with an average mid-IR enhancement of $\left\langle\epsilon_{\mathrm{MIR}}\right\rangle=4.91_{-0.39}^{+0.42}$. The presence of radio emission, $\mathrm{Na}$ I D enhancement, or morphological disruption might influence $\epsilon_{\mathrm{MIR}}$, but not in a way that significantly deviates from the underlying CO-SPOG population.

5. The enhancement in the $\mathrm{Na} I \mathrm{D}$ absorption relative to the $\mathrm{Mg} \mathrm{b}$ absorption is more significant in the CO-SPOGs than in the general SPOG population, with $19 / 52$ $(37 \% \pm 7 \%)$ detected $3 \sigma$ above the empirical relation from the original ELG sample. This may be due to the likely AGN overpopulation within the CO-SPOG sample, further supported by the larger $\mathrm{NaID}$ enhancement present in the radio-detected objects.

K.A. thanks K. Decker French for useful discussions regarding the "E+A" sample. K.A. also thanks Jonathan McDowell \& Michael J. I. Brown for Twitter dialogues regarding WISE and mid-infrared dust emission in AGNs, improving the manuscript. We also thank the anonymous referee for an insightful report that has improved the manuscript.

Support for K.A. is provided by NASA through Hubble Fellowship grant \#HST-HF2-51352.001 awarded by the Space Telescope Science Institute, which is operated by the Association of Universities for Research in Astronomy, Inc., for NASA, under contract NAS5-26555. U.L. acknowledges 

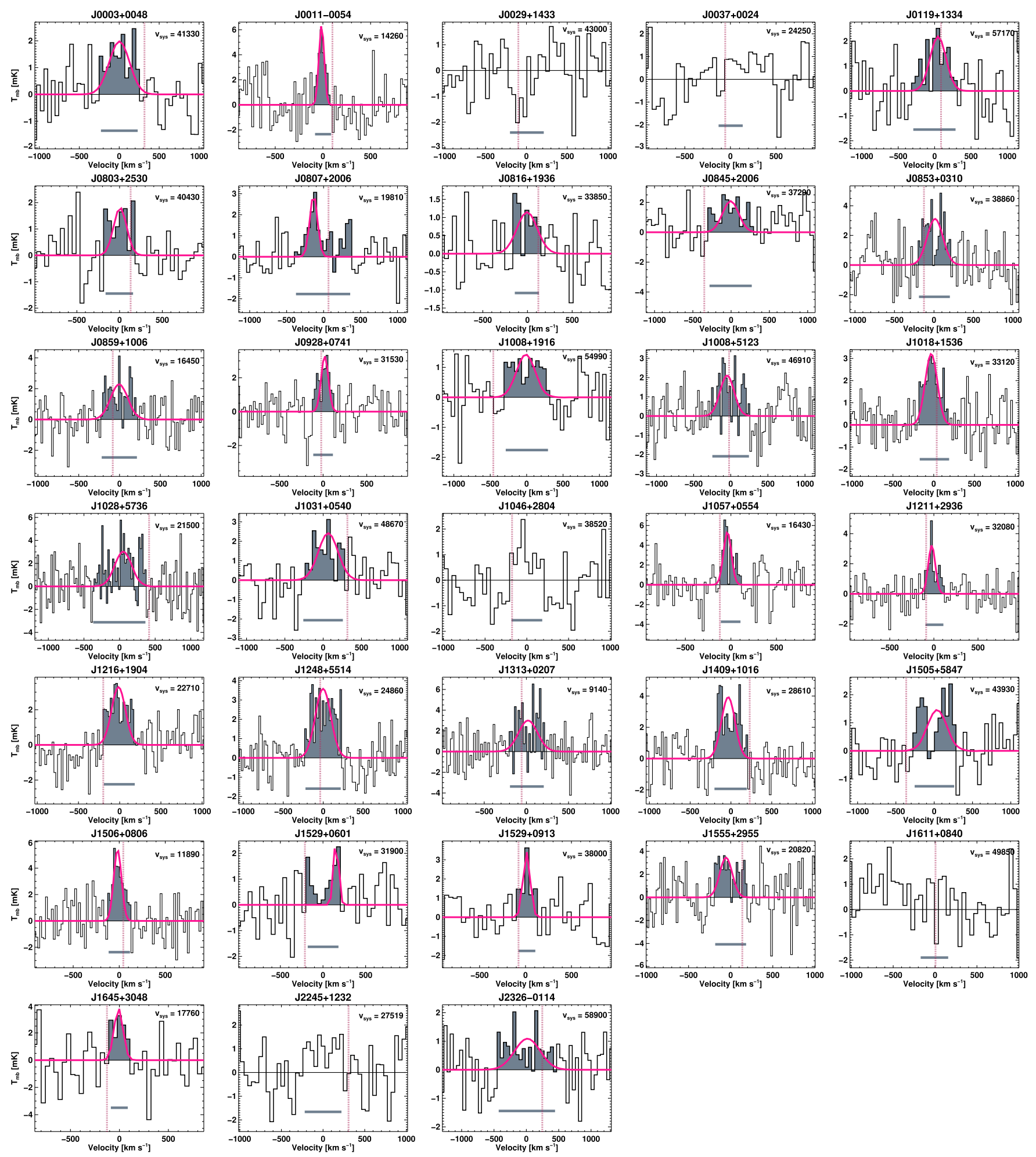

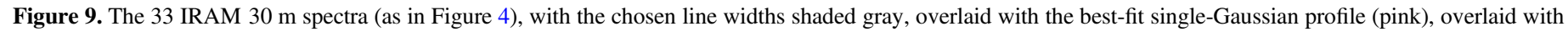
the optically determined velocity (dotted maroon line).

support by the research projects AYA2011-24728 and AYA2014-53506-P financed by the Spanish Ministerio de Economía y Competividad and by FEDER (Fondo Europeo de Desarrollo Regional) and the Junta de Andalucía (Spain) grants FQM108. P.N.A. is partially supported by funding through Herschel, a European Space Agency Cornerstone Mission with significant participation by NASA, through an award issued by JPL/Caltech. S.L.C. was supported by ALMA-CONICYT program 31110020. K.N. acknowledges support from NASA through the Spitzer Space Telescope. A.M.M. and L.J.K. acknowledge the support of the Australian Research Council (ARC) through Discovery project DP130103925. 
Based on observations carried out with the IRAM $30 \mathrm{~m}$ Telescope. IRAM is supported by INSU/CNRS (France), MPG (Germany), and IGN (Spain). Support for CARMA construction was derived from the Gordon and Betty Moore Foundation, the Kenneth T. and Eileen L. Norris Foundation, the James S. McDonnell Foundation, the Associates of the California Institute of Technology, the University of Chicago, the states of California, Illinois, and Maryland, and the National Science Foundation. Ongoing CARMA development and operations are supported by the National Science Foundation under a cooperative agreement, and by the CARMA partner universities. This publication makes use of data products from the Wide-field Infrared Survey Explorer, which is a joint project of the University of California, Los Angeles, and the Jet Propulsion Laboratory/California Institute of Technology, funded by the National Aeronautics and Space Administration. This research has made use of the NASA/ IPAC Infrared Science Archive, which is operated by the Jet Propulsion Laboratory, California Institute of Technology, under contract with the National Aeronautics and Space Administration. The National Radio Astronomy Observatory is a facility of the National Science Foundation operated under cooperative agreement by Associated Universities, Inc.

Facilities: CARMA, IRAM, WISE.

\section{APPENDIX \\ GAUSSIAN FITTING THE IRAM 30 M OBSERVATIONS}

As a check of the accuracy of the fluxes measured by the IRAM $30 \mathrm{~m}$ (especially those with $\mathrm{S} / \mathrm{N}$ between 3 and 5), we fit each spectrum to a single-Gaussian profile in order to investigate the likelihood that our detections are real or spurious. We used the minimize procedure in GILDAS ${ }^{16}$ on the continuum-subtracted, calibrated IRAM SPOG data sets (excluding nondetections). The minimize procedure was free to search for the line in a $3000 \mathrm{~km} \mathrm{~s}^{-1}$ width centered on the recession velocity, but no initial velocity guess was provided for the $\mathrm{CO}(1-0)$ line. Figure 9 shows the results of the single-Gaussian fits (pink) overlaid on the spectra of the IRAM SPOGs, with the chosen velocity widths shaded gray underlaid. The cases with a disagreement between the optical and radio recession velocities are most likely due to uncertainties in the SDSS recession velocities (individual SDSS spectral channels have widths of $\approx 100 \mathrm{~km} \mathrm{~s}^{-1}$; Bolton et al. 2012), although it is possible that the molecular gas in some of these systems is systemically offset from the stars and possibly part of a tidal tail or some disrupted gas structure, though we consider the former possibility more likely. Overall, we can see that for the majority of sources, the Gaussian fitting faithfully detected and fit the profiles, although in some cases the SPOGs would have been better fit with an additional Gaussian.

Figure 10 shows the direct comparison between the intensities derived from both methods for strong detections ( $>5 \sigma$, blue points) and tentative detections $(3 \sigma-5 \sigma$, red triangles) within the IRAM sample. In all but two cases, the flux determinations match within errors, with an average mismatch of $5 \%$. In the cases where the Gaussian method has underestimated the total intensities, often at least two Gaussian profiles were needed for the fit (e.g., J1505+5847 and $\mathrm{J} 1529+0601)$.

\footnotetext{
16 http://www.iram.fr/IRAMFR/GILDAS
}

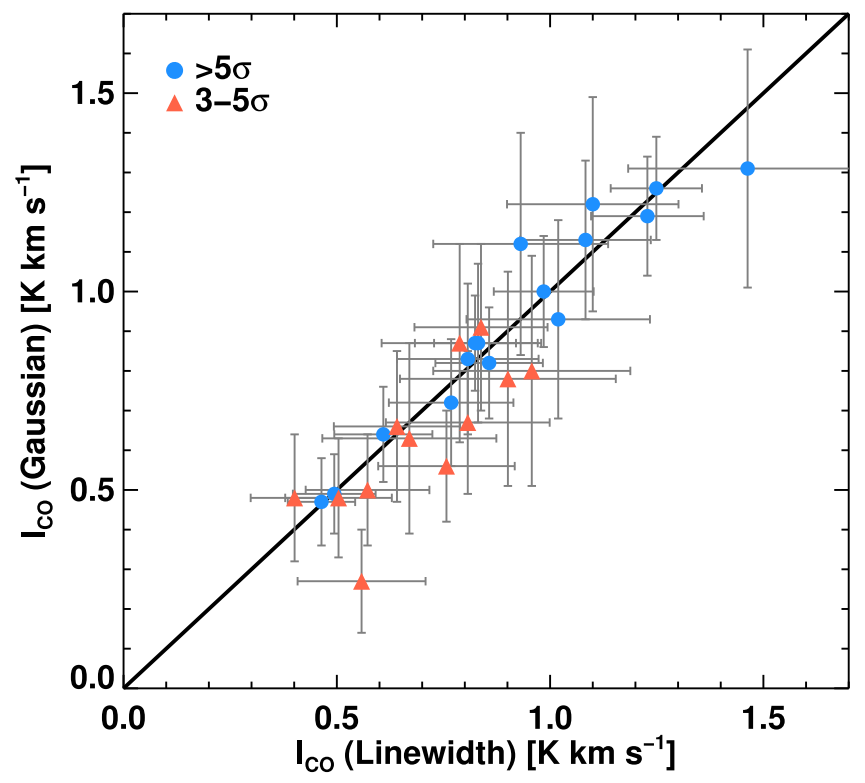

Figure 10. Comparison between $I_{\mathrm{CO}}$ determined in both of the methods: fitting a single Gaussian vs. integrating the total intensity of the line for detected IRAM CO-SPOGs. Strong $(\mathrm{S} / \mathrm{N}>5)$ detections are shown as blue circles, and tentative ones $(\mathrm{S} / \mathrm{N}=3-5)$ are shown as red triangles. The average disagreement between the two methods is $5 \%$, and the vast majority of objects agree within errors.

Overall, the Gaussian fit fluxes agreed well with our linewidth-determined fluxes, both in confirming the presence of the line at the optically defined velocity and in the total amount of flux that was detected.

\section{REFERENCES}

Aalto, S., Booth, R. S., Black, J. H., \& Johansson, L. E. B. 1995, A\&A, 300,369

Aalto, S., Costagliola, F., Muller, S., et al. 2016, A\&A, 590, A73

Aalto, S., Garcia-Burillo, S., Muller, S., et al. 2012a, A\&A, 537, A44

Aalto, S., Muller, S., Sakamoto, K., et al. 2012b, A\&A, 546, A68

Alatalo, K. 2015, ApJL, 801, L17

Alatalo, K., Appleton, P. N., Lisenfeld, U., et al. 2015a, ApJ, 812, 117

Alatalo, K., Blitz, L., Young, L. M., et al. 2011, ApJ, 735, 88

Alatalo, K., Cales, S. L., Appleton, P. N., et al. 2014a, ApJL, 794, L13

Alatalo, K., Cales, S. L., Rich, J. A., et al. 2016, ApJS, 224, 38

Alatalo, K., Crocker, A. F., Aalto, S., et al. 2015b, MNRAS, 450, 3874

Alatalo, K., Davis, T. A., Bureau, M., et al. 2013, MNRAS, 432, 1796

Alatalo, K., Lacy, M., Lanz, L., et al. 2015c, ApJ, 798, 31

Alatalo, K., Nyland, K., Graves, G., et al. 2014b, ApJ, 780, 186

Allen, M. G., Groves, B. A., Dopita, M. A., et al. 2008, ApJS, 178, 20

Appleton, P. N., Mundell, C., Bitsakis, T., et al. 2014, ApJ, 797, 117

Baade, W. 1958, RA, 5, 3

Baldry, I. K., Glazebrook, K., Brinkmann, J., et al. 2004, ApJ, 600, 681

Baldwin, J. A., Phillips, M. M., \& Terlevich, R. 1981, PASP, 93, 5

Bauermeister, A., Blitz, L., Bolatto, A., et al. 2013, ApJ, 768, 132

Becker, R. H., White, R. L., \& Helfand, D. J. 1995, ApJ, 450, 559

Bekki, K., Couch, W. J., \& Shioya, Y. 2002, ApJ, 577, 651

Bell, E. F., McIntosh, D. H., Katz, N., \& Weinberg, M. D. 2003, ApJS, 149,289

Bitsakis, T., Charmandaris, V., Appleton, P. N., et al. 2014, A\&A, 565, A25

Bitsakis, T., Charmandaris, V., da Cunha, E., et al. 2011, A\&A, 533, A142

Bitsakis, T., Charmandaris, V., Le Floc'h, E., et al. 2010, A\&A, 517, A75

Blanton, M. R., \& Moustakas, J. 2009, ARA\&A, 47, 159

Bock, D. C.-J., Bolatto, A. D., Hawkins, D. W., et al. 2006, in Proc. SPIE, 6267, 626713

Bolatto, A. D., Wolfire, M., \& Leroy, A. K. 2013, ARA\&A, 51, 207 Bolton, A. S., Schlegel, D. J., Aubourg, É., et al. 2012, AJ, 144, 144 Cales, S. L., Brotherton, M. S., Shang, Z., et al. 2011, ApJ, 741, 106 Cales, S. L., Brotherton, M. S., Shang, Z., et al. 2013, ApJ, 762, 90 Calzetti, D., Kennicutt, R. C., Engelbracht, C. W., et al. 2007, ApJ, 666, 870 Canalizo, G., \& Stockton, A. 2013, ApJ, 772, 132 
Cannon, A. J., \& Pickering, E. C. 1918, AnHar, 91, 1

Carilli, C. L., \& Walter, F. 2013, ARA\&A, 51, 105

Carter, M., Lazareff, B., Maier, D., et al. 2012, A\&A, 538, A89

Casey, C. M., Scoville, N. Z., Sanders, D. B., et al. 2014, ApJ, 796, 95

Chung, A., van Gorkom, J. H., Kenney, J. D. P., Crowl, H., \& Vollmer, B 2009, AJ, 138, 1741

Cicone, C., Feruglio, C., Maiolino, R., et al. 2012, A\&A, 543, A99

Cicone, C., Maiolino, R., Sturm, E., et al. 2014, A\&A, 562, A21

Cluver, M. E., Appleton, P. N., Ogle, P., et al. 2013, ApJ, 765, 93

Combes, F., García-Burillo, S., Braine, J., et al. 2013, A\&A, 550, A41

Combes, F., Young, L. M., \& Bureau, M. 2007, MNRAS, 377, 1795

Condon, J. J. 1992, ARA\&A, 30, 575

Crocker, A. F., Bureau, M., Young, L. M., \& Combes, F. 2011, MNRAS, 410, 1197

Crocker, A. F., Calzetti, D., Thilker, D. A., et al. 2013, ApJ, 762, 79

da Cunha, E., Charlot, S., \& Elbaz, D. 2008, MNRAS, 388, 1595

Dale, D. A., Aniano, G., Engelbracht, C. W., et al. 2012, ApJ, 745, 95

Davis, T. A., Alatalo, K., Sarzi, M., et al. 2011, MNRAS, 417, 882

Davis, T. A., Krajnović, D., McDermid, R. M., et al. 2012, MNRAS, 426, 1574

Davis, T. A., Young, L. M., Crocker, A. F., et al. 2014, MNRAS, 444, 3427

Deller, A. T., \& Middelberg, E. 2014, AJ, 147, 14

Dopita, M. A., Pereira, M., Kewley, L. J., \& Capaccioli, M. 2002, ApJS, 143,47

Downes, D., \& Solomon, P. M. 1998, ApJ, 507, 615

Draine, B. T., Dale, D. A., Bendo, G., et al. 2007, ApJ, 663, 866

Dressler, A., \& Gunn, J. E. 1983, ApJ, 270, 7

Dressler, A., Oemler, A., Jr., Poggianti, B. M., et al. 2013, ApJ, 770, 62

Elvis, M., Wilkes, B. J., McDowell, J. C., et al. 1994, ApJS, 95, 1

Evans, A. S., Mazzarella, J. M., Surace, J. A., et al. 2005, ApJS, 159, 197

Faber, S. M., Willmer, C. N. A., Wolf, C., et al. 2007, ApJ, 665, 265

Feruglio, C., Fiore, F., Carniani, S., et al. 2015, A\&A, 583, A99

Feruglio, C., Maiolino, R., Piconcelli, E., et al. 2010, A\&A, 518, L155

Fischer, J., Sturm, E., González-Alfonso, E., et al. 2010, A\&A, 518, L41

French, K. D., Yang, Y., Zabludoff, A., et al. 2015, ApJ, 801, 1

Goto, T. 2005, MNRAS, 357, 937

Goto, T. 2007, MNRAS, 377, 1222

Guillard, P., Boulanger, F., Lehnert, M. D., et al. 2015, A\&A, 574, A32

Harker, J. J., Schiavon, R. P., Weiner, B. J., \& Faber, S. M. 2006, ApJL, 647, L103

Hickson, P., Mendes de Oliveira, C., Huchra, J. P., \& Palumbo, G. G. 1992, ApJ, 399, 353

Holmberg, E. 1958, MeLuS II, 136, 1

Hopkins, P. F., Hernquist, L., Cox, T. J., et al. 2006, ApJS, 163, 1

Hubble, E. P. 1926, ApJ, 64, 321

Ivezić, Ž, Menou, K., Knapp, G. R., et al. 2002, AJ, 124, 2364

Jeong, H., Yi, S. K., Kyeong, J., et al. 2013, ApJS, 208, 7

Kaneko, H., Kuno, N., Iono, D., et al. 2014, PASJ, submitted, (arXiv:1411.2660)

Kauffmann, G., Heckman, T. M., Tremonti, C., et al. 2003, MNRAS, 346, 1055

Kenney, J. D. P., Geha, M., Jáchym, P., et al. 2014, ApJ, 780, 119

Kennicutt, R. C., Jr. 1998, ApJ, 498, 541

Kewley, L. J., Groves, B., Kauffmann, G., \& Heckman, T. 2006, MNRAS, 372,961

Ko, J., Hwang, H. S., Lee, J. C., \& Sohn, Y.-J. 2013, ApJ, 767, 90

Kocevski, D. D., Lemaux, B. C., Lubin, L. M., et al. 2011, ApJL, 737, L38

Lanz, L., Ogle, P. M., Alatalo, K., \& Appleton, P. N. 2016, ApJ, 224, 38

Laor, A., \& Behar, E. 2008, MNRAS, 390, 847

Lehnert, M. D., Tasse, C., Nesvadba, N. P. H., Best, P. N., \& van Driel, W. 2011, A\&A, 532, L3

Leon, S., Combes, F., \& Menon, T. K. 1998, A\&A, 330, 37

Lisenfeld, U., Appleton, P. N., Cluver, M. E., et al. 2014, A\&A, 570, A24

Lisenfeld, U., Espada, D., Verdes-Montenegro, L., et al. 2011, A\&A, 534, A102

Martig, M., Bournaud, F., Teyssier, R., \& Dekel, A. 2009, ApJ, 707, 250
Martig, M., Crocker, A. F., Bournaud, F., et al. 2013, MNRAS, 432, 1914

Martin, D. C., Wyder, T. K., Schiminovich, D., et al. 2007, ApJS, 173, 342

Martinez-Badenes, V., Lisenfeld, U., Espada, D., et al. 2012, A\&A, 540, A96

McBride, J., Quataert, E., Heiles, C., \& Bauermeister, A. 2014, ApJ, 780, 182

Murray, N., Martin, C. L., Quataert, E., \& Thompson, T. A. 2007, ApJ, 660,211

Narayanan, D., Krumholz, M., Ostriker, E. C., \& Hernquist, L. 2011, MNRAS, 418,664

Nyland, K., Alatalo, K., Wrobel, J. M., et al. 2013, ApJ, 779, 173

O’Connell, R. W. 1999, ARA\&A, 37, 603

Oh, K., Sarzi, M., Schawinski, K., \& Yi, S. K. 2011, ApJS, 195, 13

Park, C., Choi, Y.-Y., Vogeley, M. S., et al. 2007, ApJ, 658, 898

Park, J., Jeong, H., \& Yi, S. K. 2015, ApJ, 809, 91

Quintero, A. D., Hogg, D. W., Blanton, M. R., et al. 2004, ApJ, 602, 190

Rangwala, N., Maloney, P. R., Glenn, J., et al. 2011, ApJ, 743, 94

Rasmussen, J., Ponman, T. J., Verdes-Montenegro, L., Yun, M. S., \& Borthakur, S. 2008, MNRAS, 388, 1245

Rich, J. A., Kewley, L. J., \& Dopita, M. A. 2011, ApJ, 734, 87

Rich, J. A., Kewley, L. J., \& Dopita, M. A. 2014, ApJL, 781, L12

Rich, J. A., Kewley, L. J., \& Dopita, M. A. 2015, ApJS, 221, 28

Rosenberg, M. J. F., van der Werf, P. P., Aalto, S., et al. 2015, ApJ, 801, 72

Rowlands, K., Wild, V., Nesvadba, N., et al. 2015, MNRAS, 448, 258

Rupke, D. S., Veilleux, S., \& Sanders, D. B. 2005, ApJS, 160, 115

Rupke, D. S. N., \& Veilleux, S. 2011, ApJL, 729, L27

Rupke, D. S. N., \& Veilleux, S. 2015, ApJ, 801, 126

Saintonge, A., Kauffmann, G., Kramer, C., et al. 2011, MNRAS, 415, 32

Salomé, Q., Salomé, P., Combes, F., Hamer, S., \& Heywood, I. 2016, A\&A, 586, A45

Sanders, D. B., Phinney, E. S., Neugebauer, G., Soifer, B. T., \& Matthews, K. 1989, ApJ, 347, 29

Sandstrom, K. M., Leroy, A. K., Walter, F., et al. 2013, ApJ, 777, 5

Sarzi, M., Kaviraj, S., Nedelchev, B., et al. 2016, MNRAS, 456, L25

Sault, R. J., Teuben, P. J., \& Wright, M. C. H. 1995, in AIP Conf. Ser. 77, Astronomical Data Analysis Software and Systems IV, ed. R. A. Shaw, H. E. Payne, \& J. J. E. Hayes (Melville, NY: AIP), 433

Schawinski, K., Urry, C. M., Simmons, B. D., et al. 2014, MNRAS, 440,889

Scoville, N., Aussel, H., Sheth, K., et al. 2014, ApJ, 783, 84

Skrutskie, M. F., Cutri, R. M., Stiening, R., et al. 2006, AJ, 131, 1163

Solomon, P. M., \& Vanden Bout, P. A. 2005, ARA\&A, 43, 677

Spergel, D. N., Bean, R., Doré, O., et al. 2007, ApJS, 170, 377

Springel, V., Di Matteo, T., \& Hernquist, L. 2005, ApJL, 620, L79

Strateva, I., Ivezić, Ž, Knapp, G. R., et al. 2001, AJ, 122, 1861

Sturm, E., González-Alfonso, E., Veilleux, S., et al. 2011, ApJL, 733, L16

Tinsley, B. M. 1978, ApJ, 222, 14

Toomre, A., \& Toomre, J. 1972, ApJ, 178, 623

Vazdekis, A., Sánchez-Blázquez, P., Falcón-Barroso, J., et al. 2010, MNRAS, 404, 1639

Veilleux, S., Cecil, G., \& Bland-Hawthorn, J. 2005, ARA\&A, 43, 769

Veilleux, S., \& Osterbrock, D. E. 1987, ApJS, 63, 295

Verdes-Montenegro, L., Yun, M. S., Perea, J., del Olmo, A., \& Ho, P. T. P. 1998, ApJ, 497, 89

Ward, M., Elvis, M., Fabbiano, G., et al. 1987, ApJ, 315, 74

Wild, V., Charlot, S., Brinchmann, J., et al. 2011, MNRAS, 417, 1760

Wild, V., Walcher, C. J., Johansson, P. H., et al. 2009, MNRAS, 395, 144

Wright, E. L., Eisenhardt, P. R. M., Mainzer, A. K., et al. 2010, AJ, 140, 1868

Yan, R., Newman, J. A., Faber, S. M., et al. 2006, ApJ, 648, 281

Yao, L., Seaquist, E. R., Kuno, N., \& Dunne, L. 2003, ApJ, 588, 771

Yesuf, H. M., Faber, S. M., Trump, J. R., et al. 2014, ApJ, 792, 84

Young, L. M., Bureau, M., Davis, T. A., et al. 2011, MNRAS, 414, 940

Young, L. M., Scott, N., Serra, P., et al. 2014, MNRAS, 444, 3408

Zabludoff, A. I., \& Mulchaey, J. S. 1998, ApJ, 496, 39

Zabludoff, A. I., Zaritsky, D., Lin, H., et al. 1996, ApJ, 466, 104

Zensus, J. A. 1997, ARA\&A, 35, 607

Zucker, C., Walker, L. M., Johnson, K., et al. 2016, ApJ, 821, 113 